\title{
A Spatial Model of Tumor Growth with Cell Age, Cell Size, and Mutation of Cell Phenotypes
}

\author{
J. Dyson ${ }^{a}$, R.Villella-Bressan ${ }^{b}$ and G. Webb ${ }^{c} 12$ \\ ${ }^{a}$ Mansfield College, University of Oxford, Oxford, England \\ ${ }^{b}$ Dipartimento di Matematica Pura e Applicata, Universita di Padova, Padova, Italy \\ ${ }^{c}$ Department of Mathematics, Vanderbilt University, Nashville, Tennessee, USA
}

\begin{abstract}
A model of tumor growth in a spatial environment is analyzed. The model includes proliferating and quiescent compartments of tumor cells indexed by successively mutated cell phenotypes of increasingly proliferative aggressiveness. The model incorporates spatial dependence due to both random motility and directed movement haptotaxis. The model structures tumor cells by both cell age and cell size. The model consists of a system of nonlinear partial differential equations for the compartments of tumor cells, extracellular matrix, matrix degradative enzyme, and oxygen. The existence, uniqueness, positivity, regularity, and growth characteristics of the solutions are investigated.
\end{abstract}

Key words: tumor growth, haptotaxis, analytic semigroups, nonlinear partial differential equations AMS subject classification: $92 \mathrm{C} 17$, 92D25, 35K45

\section{Introduction}

Mathematical models of tumor growth in spatial environments confront the unique characteristics of the complex biological processes involved. A tumor mass consists of individual cells rather than dimensionless particles or continuum fluid, and the heterogeneity of individual cell growth and division is a fundamental consideration of the growth of the total tumor mass. There is an extensive literature of spatial models of tumor growth that have included many of these processes in both continuum and discrete frameworks. Our objective here is to continue the study of a

\footnotetext{
${ }^{1}$ Corresponding author. E-mail: glenn.f.webb@vanderbilt.edu

${ }^{2}$ Research of the third author is supported by PHS - NIH Grant \#1P50CA113007-01
} 
continuum model of tumor growth first proposed in [5] (see also [3] and [4]), and later developed in [6], [7], [8], [18], and [19]. The salient feature of this model is the use of cell age to track passage of individual cells through the cell cycle. In [6], [7] a basic theory of existence, uniqueness, positivity, and regularity of solutions was established for the system of nonlinear partial differential equations of the model. A major difficulty in the analysis was to treat the term corresponding to haptotaxis, the directed movement of tumor cells up-gradient of the environmental extracellular matrix, as mediated by a degradation enzyme produced by the tumor cells. We assume a nonlocal effect for this process, that is, extracellular macromolecules interact with tumor cells in a nearby region (a local assumption for this effect was analyzed in [18] and [19]).

In this paper we extend the model in [6], [7] to include cell size as a structure variable analogous to cell age, which allows consideration of individual cell growth and division as a component of the spatial movement of the total tumor mass. Additionally, we include transition of cells to and from quiescence as a constraint on tumor growth limited by availability of oxygen and other nutrients. Lastly, we include mutations that confer proliferative advantage to successive phenotypic cell lines. An important goal of tumor modeling is to verify the consistency of these mutation rates with the time scale of clonal phenotype succession through large numbers of possible somatic mutations in cancer genomes ([9]).

The organization of this paper is as follows: In Section 3 we give some results from other papers which will be required in the sequel and we also establish the properties of the semigroup $\{S(t)\}_{t \geq 0}$ of the linear problem associated with the full nonlinear problem. In Section 4 we establish the local existence of a mild solution, and then in Section 5 show that there is in fact a unique global mild solution, which has continuous dependence on the initial data. In Sections 6 and 7 we concentrate on the problem without quiescence, establishing regularity and positivity results. In Section 8 we return to the problem with quiescence and, by considering it as a perturbation of the problem without quiescence, show that in this case we also have positivity. In Section 9 a growth bound is established in the case where there is no quiescence and solutions are positive. Then in Section 10 we return to the case where there is quiescence. We show that if the cell age and size ranges are infinite, we have regularity for the solutions; we then give a growth bound when solutions are positive. Lastly, in Section 11 we provide numerical examples to illustrate the role of haptotaxis in the spatial development of tumor growth, as well as the advantage gained by phenotype mutation to greater proliferative capacity.

\section{Equations of the Model}

The model we investigate is discussed in detail in [5]. The spatial variable $x$ is contained in a spatial region $\Omega \subset \mathbb{R}^{n}, n=1,2,3$, where $\Omega$ is a non-empty bounded open set with smooth boundary $\partial \Omega$. The age variable $a$ is contained in $\left[0, a_{1}\right]$, and the size variable $s$ is contained in $\left[0, s_{1}\right]$. The model consists of the following compartments:

- $f(x, t)=$ surrounding tissue macromolecule (MM) density at position $x$ at time $t$.

- $m(x, t)=$ matrix degradative enzyme (MDE) concentration at position $x$ at time $t$. 
- $w(x, t)=$ oxygen concentration at position $x$ at time $t$.

- $p_{i}(x, a, s, t)=$ proliferating tumor cells of type $i$ in the tumor at position $x$, age $a$, and size $s$ at time $t$, where $i=0,1,2, \ldots, n$ corresponds to a linear sequence of mutated phenotypes of increasing aggressiveness.

- $q_{i}(x, a, s, t)=$ quiescent tumor cells of type $i$ in the tumor at position $x$, age $a$, and size $s$ at time $t$. Quiescent cells are immobile and do not age.

Let $P_{i}(x, t)=\int_{0}^{a_{1}} \int_{0}^{s_{1}} p_{i}(x, a, s, t) \mathrm{d} s \mathrm{~d} a, P(x, t)=\sum_{i=0}^{n} P_{i}(x, t)=$ the total population density at $x$ of proliferating cells of all types at time $t$. Let $Q_{i}(x, t)=\int_{0}^{a_{1}} \int_{0}^{s_{1}} q_{i}(x, a, s, t) \mathrm{d} s \mathrm{~d} a, Q(x, t)=$ $\sum_{i=0}^{n} Q_{i}(x, t)=$ the total population density at $x$ of quiescent cells of all types at time $t$ and let $N(x, t)=P(x, t)+Q(x, t)$. The equations of the model are as follows:

$$
\begin{aligned}
& \frac{\partial}{\partial t} f(x, t)=\underbrace{\delta_{f} \nabla^{2} f(x, t)}_{\text {motility }}-\underbrace{\omega_{f} f(x, t)}_{\text {decay }}-\underbrace{\lambda_{f}(x, m(\cdot, t), w(\cdot, t), P(\cdot, t)) f(x, t)}_{\text {degradation }}, \\
& \frac{\partial}{\partial t} m(x, t)=\underbrace{\delta_{m} \nabla^{2} m(x, t)}_{\text {diffusion }}-\underbrace{\omega_{m} m(x, t)}_{\text {decay }}+\underbrace{\rho_{m}(x, P(\cdot, t)) P(x, t)}_{\text {production }}, \\
& \frac{\partial}{\partial t} w(x, t)=\underbrace{\delta_{w} \nabla^{2} w(x, t)}_{\text {diffusion }}-\underbrace{\omega_{w} w(x, t)}_{\text {decay }}+\underbrace{\beta_{w}(x, f(\cdot, t)) f(x, t)}_{\text {production }} \\
& -\underbrace{\mu_{w}(x, w(\cdot, t), P(\cdot, t), Q(\cdot, t)) w(x, t)}_{\text {up-take }}, \\
& \frac{\partial}{\partial t} p_{i}(x, a, s, t)=-\underbrace{\frac{\partial}{\partial a} p_{i}(x, a, s, t)}_{\text {cell aging }}-\underbrace{\frac{\partial}{\partial s}\left(\eta_{i}(s) p_{i}(x, a, s, t)\right)}_{\text {cell growth }}+\underbrace{\left.\delta_{i} \nabla^{2} p_{i}(x, a, s, t)\right)}_{\text {motility }} \\
& -\underbrace{\omega p_{i}(x, a, s, t)}_{\text {cell loss }}-\underbrace{\chi_{i} \nabla \cdot\left(p_{i}(x, a, s, t) \nabla f(x, t)\right)}_{\text {haptotaxis }} \\
& -\underbrace{\mu_{i}(x, a, s, w(\cdot, t), N(\cdot, t)) p_{i}(x, a, s, t)}_{\text {cell loss from division or insufficient oxygen }} \\
& -\underbrace{\sigma_{i}(x, a, s, w(\cdot, t), N(\cdot, t)) p_{i}(x, a, s, t)}_{\text {exit to quiescence }} \\
& +\underbrace{\tau_{i}(x, a, s, w(\cdot, t), N(\cdot, t)) q_{i}(x, a, s, t)}_{\text {entry from quiescence }},
\end{aligned}
$$




$$
\begin{aligned}
\frac{\partial}{\partial t} q_{i}(x, a, s, t)= & -\underbrace{\nu_{i}(x, a, s, w(\cdot, t), N(\cdot, t)) q_{i}(x, a, s, t)}_{\text {cell death from insufficient oxygen }} \\
& +\underbrace{\sigma_{i}(x, a, s, w(\cdot, t), N(\cdot, t)) p_{i}(x, a, s, t)}_{\text {entry from proliferation }} \\
& -\underbrace{\tau_{i}(x, a, s, w(\cdot, t), N(\cdot, t)) q_{i}(x, a, s, t)}_{\text {exit to proliferation }}
\end{aligned}
$$

We set the age and size boundaries at cell birth to

$$
\begin{gathered}
p_{i}(x, 0, s, t)=2\left(1-\psi_{i}\right) \int_{0}^{a_{1}} \int_{0}^{s_{1}} \kappa_{i}(\hat{s}, s) \beta_{i}(\hat{a}, \hat{s}) p_{i}(x, \hat{a}, \hat{s}, t) \mathrm{d} \hat{s} \mathrm{~d} \hat{a}+ \\
2 \psi_{i-1} \int_{0}^{a_{1}} \int_{0}^{s_{1}} \kappa_{i-1}(\hat{s}, s) \beta_{i-1}(\hat{a}, \hat{s}) p_{i-1}(x, \hat{a}, \hat{s}, t) \mathrm{d} \hat{s} \mathrm{~d} \hat{a} \\
p_{i}(x, a, 0, t)=0, a \in\left(0, a_{1}\right),
\end{gathered}
$$

where $\psi_{i}$ is the $i^{\text {th }}$ phenotype mutation rate, and $\psi_{-1}=0$. We take Neumann boundary conditions

$$
\left.\frac{\partial f(x, t)}{\partial n}\right|_{\partial \Omega}=0,\left.\quad \frac{\partial m(x, t)}{\partial n}\right|_{\partial \Omega}=0,\left.\quad \frac{\partial w(x, t)}{\partial n}\right|_{\partial \Omega}=0,
$$

and

and initial conditions

$$
\left.\frac{\left.\partial p_{i}(x, a, s, t)\right)}{\partial n}\right|_{\partial \Omega}=0
$$

$$
f(\cdot, 0)=f_{0}, \quad m(\cdot, 0)=m_{0}, \quad w(\cdot, 0)=w_{0}, \quad p_{i}(\cdot, \cdot, \cdot, 0)=\phi_{i}, \quad q_{i}(\cdot, \cdot, \cdot, 0)=\zeta_{i} .
$$

We assume that the constants $\delta_{f}>0, \delta_{m}>0, \delta_{w}>0, \delta_{i}>0, \chi_{i}>0, \omega_{f}>0, \omega_{m} \geq 0$, $\omega_{w} \geq 0, \omega>0,0 \leq \psi_{i}<1$. For simplicity we set $\eta_{i}(s)=1$, which can be achieved in many cases with a simple transformation of $s$ ([23]). We will set $\theta_{i}(\hat{a}, \hat{s}, s)=2\left(1-\psi_{i}\right) \kappa_{i}(\hat{s}, s) \beta_{i}(\hat{a}, \hat{s}) \geq 0$.

\section{Preliminaries}

We denote by $\|\cdot\|_{r}$ the norm in $L^{r}(\Omega)$, by $\|\cdot\|_{\infty}$ the norm in $C(\bar{\Omega})$ and by $\|\cdot\|_{s, r}$ the norm in the Sobolev(-Slobodeckii) space $W^{s, r}(\Omega)$. Let $W^{2, r}(\Omega ; B)=\left\{u \in W^{2, r}(\Omega):\left.B u\right|_{\partial \Omega}=0\right\}$, where $B u=\frac{\partial u}{\partial n}$, the normal derivative. We will consider the realizations of the Laplacian in different Banach spaces. Define the operator $A_{i}: D\left(A_{i}\right) \subset L^{1}(\Omega) \rightarrow L^{1}(\Omega)$ as the closure in $L^{1}(\Omega)$ of the operator $A_{i, r}$ where, for any $1<r<\infty, A_{i, r}: D\left(A_{i, r}\right) \subset L^{r}(\Omega) \rightarrow L^{r}(\Omega)$ is defined by

$$
\begin{aligned}
A_{i, r} u & =\left(-\delta_{i} \Delta+\omega\right) u, u \in D\left(A_{i, r}\right), \quad \text { with } \\
D\left(A_{i, r}\right) & =W^{2, r}(\Omega ; B) .
\end{aligned}
$$


We define the unbounded operators $A_{m}, A_{w}$ from $D\left(A_{m}\right)$ and $D\left(A_{w}\right)$ to $L^{1}(\Omega)$ similarly. It is well known (see [1]) that $-A_{i}$ generates a positive analytic semigroup $\left\{T_{i}(t)\right\}_{t \geq 0}$, with $\left\|T_{i}(t)\right\| \leq$ $e^{-\omega t}$ and similarly $-A_{m},-A_{w}$ generate positive analytic semigroups $\left\{T_{m}(t)\right\}_{t \geq 0},\left\{T_{w}(t)\right\}_{t \geq 0}$, with $\left\|T_{m}(t)\right\| \leq e^{-\omega_{m} t}$ and $\left\|T_{w}(t)\right\| \leq e^{-\omega_{w} t}$ (where \|\| is the operator norm).

We define $A_{f}: D\left(A_{f}\right) \subset C(\bar{\Omega}) \rightarrow C(\bar{\Omega})$, by

$$
A_{f} u=\left(-\delta_{f} \Delta+\omega_{f}\right) u, \quad D\left(A_{f}\right)=\left\{u \in \bigcap_{1 \leq q<\infty} W^{2, q}(\Omega): \Delta u \in C(\bar{\Omega}) \text { and }\left.B u\right|_{\partial \Omega}=0\right\} .
$$

Then $-A_{f}$ generates a positive analytic semigroup $\left\{T_{f}(t)\right\}_{t \geq 0}$, with $\left\|T_{f}(t)\right\| \leq M_{f} e^{-\omega_{f} t}$ (see [1] [10], [14], [15]). We note that 0 is in the resolvent set of $A_{i}$ and also of $A_{f}$.

We will use the fractional powers $A_{i}^{\alpha}$ of $A_{i}$. We have, from [13] theorem 2.6.13 and lemma 2.6.3:

$\mathrm{i}$ if $\alpha>0$, for every $u \in D\left(A_{i}^{\alpha}\right), T_{i}(t) A_{i}^{\alpha} u=A_{i}^{\alpha} T_{i}(t) u$;

ii if $\alpha>0$, for every $t>0$ the operator $A_{i}^{\alpha} T_{i}(t)$ is a bounded linear operator in $L^{1}(\Omega)$ and there exists a constant $M_{\alpha}>0$ such that

$$
\left\|A_{i}^{\alpha} T_{i}(t)\right\| \leq M_{\alpha} t^{-\alpha} e^{-\omega t}
$$

iii if $0<\alpha \leq 1$ then there exists $N_{\alpha}>0$ such that for all $u \in D\left(A_{i}^{\alpha}\right)$

$$
\left\|T_{i}(t) u-u\right\|_{1} \leq N_{\alpha} t^{\alpha}\left\|A_{i}^{\alpha} u\right\|_{1}
$$

iv if $0 \leq \alpha \leq 1$, then $A_{i}^{-\alpha}$ is a bounded linear operator in $L^{1}(\Omega)$ and there exists a constant $H \geq 1$ such that for all $u \in L^{1}(\Omega)$

$$
\left\|A_{i}^{-\alpha} u\right\|_{1} \leq H\|u\|_{1} .
$$

Note that for $0<\alpha \leq 1, D\left(A_{i}^{\alpha}\right)$ and $D\left(A_{i, r}^{\alpha}\right)$ are independent of $i$ and there exists a constant $h_{\alpha}>0$ such that $\left\|A_{j}^{\alpha} \phi\right\|_{1} \leq h_{\alpha}\left\|A_{i}^{\alpha} \phi\right\|_{1}$ for all $\phi \in D\left(A_{i}^{\alpha}\right)$, all $i, j$, and similarly for $A_{i, r}^{\alpha}$, see [11] Lemma 4.1.11.

We define, for $k=1,2,3, B_{k}: D\left(B_{k}\right) \subset L^{1}(\Omega) \rightarrow L^{1}(\Omega)$,

$$
B_{k} u=\frac{\partial u}{\partial x_{k}}, \text { with } D\left(B_{k}\right)=W^{1,1}(\Omega),
$$

so $B_{k}$ is a closed linear operator. As in [6] Lemma 1 , if $0<\beta<\alpha<1$, then $D\left(A_{i}^{\alpha}\right) \hookrightarrow W^{2 \beta, 1}(\Omega)$ and $D\left(A_{i, r}^{\alpha}\right) \hookrightarrow W^{2 \beta, r}(\Omega)$, where ' $\hookrightarrow$ ' denotes continuous embedding and if $\frac{1}{2}<\alpha<1, B_{k} A_{i}^{-\alpha}$ and $B_{k} A_{i, r}^{-\alpha}$ are bounded linear operators in $L^{1}(\Omega)$ and $L^{r}(\Omega)$ respectively (see also [16] and [17]).

Let $X=L^{1}\left(\left(0, a_{1}\right) \times\left(0, s_{1}\right) ; L^{1}(\Omega)\right) ; X_{\alpha, i}=L^{1}\left(\left(0, a_{1}\right) \times\left(0, s_{1}\right) ; D\left(A_{i}^{\alpha}\right)\right)$, endowed with the norm $\|\cdot\|_{X_{\alpha, i}}=\left\|A_{i}^{\alpha} \cdot\right\|_{X} ; X_{\alpha}=\prod_{i=0}^{n} X_{\alpha, i}$ with norm $\|\cdot\|_{X_{\alpha}}=\sum_{i=0}^{n}\|\cdot\|_{X_{\alpha, i}}$. Let $X^{n+1}$ have 
norm $\|\cdot\|_{X^{n+1}}=\sum_{i=0}^{n}\|\cdot\|_{X} ; Z$ be the space $D\left(A_{f}\right) \times L^{1}(\Omega)^{2} \times X^{2(n+1)}$ endowed with the norm $\left\|A_{f} \cdot\right\|_{\infty}+\|\cdot\|_{1}+\|\cdot\|_{1}+\|\cdot\|_{X^{n+1}}+\|\cdot\|_{X^{n+1}} ; Z_{\alpha, p}=D\left(A_{f}\right) \times L^{1}(\Omega)^{2} \times X_{\alpha}$ with norm $\left\|A_{f} \cdot\right\|_{\infty}+\|\cdot\|_{1}+\|\cdot\|_{1}+\|\cdot\|_{X_{\alpha}} ; Z_{\alpha}=Z_{\alpha, p} \times X^{n+1}$ with norm $\|\cdot\|_{Z_{\alpha, p}}+\|\cdot\|_{X^{n+1}}$.

We will assume that

$\mathrm{H}(3 . .1) \theta_{i} \in C\left(\left[0, a_{1}\right] \times\left[0, s_{1}\right] \times\left[0, s_{1}\right] ; \mathbb{R}^{+}\right)$, and if

$$
\bar{\theta}_{i}:=\sup _{\hat{a} \in\left[0, a_{1}\right], \hat{s} \in\left[0, s_{1}\right]} \int_{0}^{s_{1}} \theta_{i}(\hat{a}, \hat{s}, s) \mathrm{d} s,
$$

then $\bar{\theta}_{i}$ is strictly increasing with $i$.

Remark. This is for the case when $a_{1}$ and $s_{1}$ are finite. The results also hold in the infinite case if we substitute hypothesis $\mathrm{H}\left(3.1^{\prime}\right)$ for $\mathrm{H}(3 . .1)$, see Section 10 ..

We consider the linear system, for $a \in\left(0, a_{1}\right), s \in\left(0, s_{1}\right), t>0, \phi_{i} \in X, 0 \leq i \leq n$,

$$
\begin{aligned}
& \frac{\partial}{\partial t} p_{i}(a, s, t)+\frac{\partial}{\partial a} p_{i}(a, s, t)+\frac{\partial}{\partial s} p_{i}(a, s, t)=A_{i} p_{i}(a, s, t), \\
p_{i}(0, s, t)= & \int_{0}^{a_{1}} \int_{0}^{s_{1}} \theta_{i}(\hat{a}, \hat{s}, s) p_{i}(\hat{a}, \hat{s}, t) \mathrm{d} \hat{s} \mathrm{~d} \hat{a}+ \\
& \frac{\psi_{i-1}}{1-\psi_{i-1}} \int_{0}^{a_{1}} \int_{0}^{s_{1}} \theta_{i-1}(\hat{a}, \hat{s}, s) p_{i-1}(\hat{a}, \hat{s}, t) \mathrm{d} \hat{s} \mathrm{~d} \hat{a}, \\
p_{i}(a, s, 0)= & \phi_{i}(a, s), \quad p_{i}(a, 0, t)=0 .
\end{aligned}
$$

We will write $\phi^{i}=\left[\phi_{0}, \ldots, \phi_{i}\right]^{t}$. Throughout when $i=n$ we suppress $n$ so that $\phi:=\phi^{n}$ etc. Motivated by the method of characteristics, we associate with the solutions of the system (3.5)(3.7) the following semigroup $\{S(t)\}_{t \geq 0}$. We define the operators $S^{i}(t): X^{i+1} \rightarrow X^{i+1}$ as follows: $S^{i}(t) \phi^{i}=\left[S_{0}(t) \phi^{0}, S_{1}(t) \phi^{1}, \cdots, S_{i}(t) \phi^{i}\right]^{t}, S(t) \phi:=S^{n}(t) \phi^{n}$, where for $t=0, S_{i}(0)=I$, and for $t>0$

$$
\left(S_{i}(t) \phi^{i}\right)(a, s)= \begin{cases}T_{i}(t) \phi_{i}(a-t, s-t), & 0<t<a, 0<t<s \\ T_{i}(a) b_{i}\left(\phi^{i}\right)(s-a, t-a), & 0 \leq a<t, 0 \leq a<s \\ 0, & 0 \leq s<t, 0 \leq s<a\end{cases}
$$

Here $b_{i}\left(\phi^{i}\right)(s, t)$ satisfies

$$
\begin{aligned}
& b_{i}\left(\phi^{i}\right)(s, t)=\int_{0}^{\min \left\{t, a_{1}\right\}} \int_{\hat{a}}^{s_{1}} \theta_{i}(\hat{a}, \hat{s}, s) T_{i}(\hat{a}) b_{i}\left(\phi^{i}\right)(\hat{s}-\hat{a}, t-\hat{a}) \mathrm{d} \hat{s} \mathrm{~d} \hat{a} \\
& \quad+\Lambda \int_{t}^{a_{1}} \int_{t}^{s_{1}} \theta_{i}(\hat{a}, \hat{s}, s) T_{i}(t) \phi_{i}(\hat{a}-t, \hat{s}-t) \mathrm{d} \hat{s} \mathrm{~d} \hat{a} \\
& \quad+\frac{\psi_{i-1}}{1-\psi_{i-1}}\left(\int_{0}^{\min \left\{t, a_{1}\right\}} \int_{\hat{a}}^{s_{1}} \theta_{i-1}(\hat{a}, \hat{s}, s) T_{i-1}(\hat{a}) b_{i-1}\left(\phi^{i-1}\right)(\hat{s}-\hat{a}, t-\hat{a}) \mathrm{d} \hat{s} \mathrm{~d} \hat{a}\right. \\
& \left.+\Lambda \int_{t}^{a_{1}} \int_{t}^{s_{1}} \theta_{i-1}(\hat{a}, \hat{s}, s) T_{i-1}(t) \phi_{i-1}(\hat{a}-t, \hat{s}-t) \mathrm{d} \hat{s} \mathrm{~d} \hat{a}\right)
\end{aligned}
$$


where $\Lambda=1$ if $t<a_{1}$ and $t<s_{1}$, but $\Lambda=0$ otherwise. As in [23], for any $T>0$, this set of equations has a unique solution $b_{i}\left(\phi^{i}\right) \in C\left(\left[0, s_{1}\right] \times[0, T] ; L^{1}(\Omega)\right)$. Set $b^{i}\left(\phi^{i}\right)(s, t)=$ $\left[b_{0}\left(\phi^{0}\right)(s, t), \cdots, b_{i}\left(\phi^{i}\right)(s, t)\right]^{t}, b(\phi)(t, s):=b^{n}\left(\phi^{n}\right)(t, s)$.

From (3.8), if $0 \leq a<t, 0 \leq a<s$, then for all $\phi \in X^{n+1}$,

$$
\left(S_{i}(t) \phi^{i}\right)(a, s)=T_{i}(a)\left(b_{i}\left(\phi^{i}\right)(s-a, t-a)\right)=T_{i}(a)\left(S_{i}(t-a) \phi^{i}\right)(0, s-a),
$$

so in this region $(S(t) \phi)(a, s)$ is continuous in $(a, s)$ as $b(\phi)(s, t)$ is continuous in $(s, t)$. If in addition $\phi$ is continuous in $(a, s)$, then $(S(t) \phi)(a, s)$ is continuous for all $(a, s)$ except possibly across $a=t \leq s, a=s<t$ and $s=t<a$. If $a=t<s$ it is continuous if, for all $i$ and $s$,

$$
\begin{aligned}
\phi_{i}(0, s)= & \int_{0}^{a_{1}} \int_{0}^{s_{1}} \theta_{i}(\hat{a}, \hat{s}, s) \phi_{i}(\hat{a}, \hat{s}) \mathrm{d} \hat{s} \mathrm{~d} \hat{a} \\
& +\frac{\psi_{i-1}}{1-\psi_{i-1}}\left(\int_{0}^{a_{1}} \int_{0}^{s_{1}} \theta_{i-1}(\hat{a}, \hat{s}, s) \phi_{i-1}(\hat{a}, \hat{s}) \mathrm{d} \hat{s} \mathrm{~d} \hat{a}\right),
\end{aligned}
$$

across $a=s<t$ it is continuous if, for all $i, \theta_{i}(\cdot, \cdot, 0)=0$, and across $s=t<a$ it is continuous if $\phi(\cdot, 0)=0$. It is continuous at $a=s=t$ if $\phi(0,0)=0$ and for all $i, \theta_{i}(\cdot, \cdot, 0)=0$. Also, from (3.8) and (3.9), if $t>0$,

$$
\begin{aligned}
\left(S_{i}(t) \phi^{i}\right)(0, s)= & \int_{0}^{a_{1}} \int_{0}^{s_{1}} \theta_{i}(\hat{a}, \hat{s}, s)\left(S_{i}(t) \phi^{i}\right)(\hat{a}, \hat{s}) \mathrm{d} \hat{s} \mathrm{~d} \hat{a}+ \\
& \frac{\psi_{i-1}}{1-\psi_{i-1}} \int_{0}^{a_{1}} \int_{0}^{s_{1}} \theta_{i-1}(\hat{a}, \hat{s}, s)\left(S_{i-1}(t) \phi^{i-1}\right)(\hat{a}, \hat{s}) \mathrm{d} \hat{s} \mathrm{~d} \hat{a} .
\end{aligned}
$$

We define $T(t): L^{1}(\Omega)^{n+1} \rightarrow L^{1}(\Omega)^{n+1}, T(t) \phi=\left[T_{0}(t) \phi_{1}, \cdots, T_{n}(t) \phi_{n}\right]^{t}$. Then $T(t)$ is a strongly continuous analytic semigroup of bounded linear positive operators in $L^{1}(\Omega)^{n+1}$, with generator $A: D(A) \subset L^{1}(\Omega)^{n+1} \rightarrow L^{1}(\Omega)^{n+1}, D(A)=\prod_{i=0}^{n} D\left(A_{i}\right)$,

$$
A \phi=\left[A_{0} \phi_{0}, \cdots, A_{n} \phi_{n}\right]^{t} .
$$

Also $\|T(t)\| \leq e^{-\omega t}$ and if $\alpha>0$, for every $t>0$ the operator $A^{\alpha} T(t)$ is a bounded linear operator in $L^{1}(\Omega)^{n+1}$ and

$$
\left\|A^{\alpha} T(t)\right\| \leq M_{\alpha} t^{-\alpha} e^{-\omega t}
$$

Then from (3.8)

$$
(S(t) \phi)(a, s)= \begin{cases}T(t) \phi(a-t, s-t), & 0<t<a, 0<t<s \\ T(a) b(\phi)(s-a, t-a), & 0 \leq a<t, 0 \leq a<s \\ 0, & 0 \leq s<t, 0 \leq s<a\end{cases}
$$

Now

Lemma 1. $\{S(t)\}_{t \geq 0}$ is a strongly continuous semigroup of bounded linear positive operators in $X^{n+1}$, satisfying

$$
\left\|S_{i}(t) \phi^{i}\right\|_{X} \leq c_{i}\left(\phi^{i}\right) e^{\left(\bar{\theta}_{i}-\omega\right) t}, i \geq 0
$$


where $c_{0}\left(\phi^{0}\right)=\left\|\phi_{0}\right\|_{X}$ and for $i \geq 1$,

$$
c_{i}\left(\phi^{i}\right)=\left\|\phi_{i}\right\|_{X}+\sum_{k=0}^{i-1}\left\{\Pi_{j=k}^{i-1} \frac{\psi_{j} \bar{\theta}_{j}}{\left(1-\psi_{j}\right)\left(\bar{\theta}_{j+1}-\bar{\theta}_{j}\right)}\right\}\left\|\phi_{k}\right\|_{X} .
$$

Thus for all $\phi \in X^{n+1}, t \geq 0$,

$$
\|S(t) \phi\|_{X^{n+1}} \leq M e^{\left(\bar{\theta}_{n}-\omega\right) t}\|\phi\|_{X^{n+1}},
$$

where

$$
M=\max _{0 \leq k \leq n-1}\left\{\sum_{i=k+1}^{n}\left(\prod_{j=k}^{i-1} \frac{\psi_{j} \bar{\theta}_{j}}{\left(1-\psi_{j}\right)\left(\bar{\theta}_{j+1}-\bar{\theta}_{j}\right)}\right)\right\}+1 .
$$

Further, if $\alpha>0$, for every $t>0$ the operator $A^{\alpha} S(t)$ is a bounded linear operator in $X^{n+1}$ and there exists a constant $C_{\alpha}>0$ such that

$$
\left\|A^{\alpha} S(t)\right\| \leq \Lambda M_{\alpha} t^{-\alpha} e^{-\omega t}+C_{\alpha} e^{\left(\bar{\theta}_{n}-\omega\right) t}
$$

where $\Lambda=1$ if $t<a_{1}$ and $t<s_{1}$, but $\Lambda=0$ otherwise.

Proof. That $\{S(t)\}_{t \geq 0}$ is a strongly continuous semigroup of linear positive operators in $X^{n+1}$ follows as in [23] using induction on $i$.

Now set $g_{i}(t)=\int_{0}^{s_{1}}\left\|b_{i}\left(\phi^{i}\right)(s, t)\right\|_{1} \mathrm{~d} s$. We show first that

$$
g_{i}(t) \leq \bar{\theta}_{i} c_{i}\left(\phi^{i}\right) e^{\left(\bar{\theta}_{i}-\omega\right) t} .
$$

From (3.9)

$$
\begin{aligned}
g_{i}(t) \leq & \bar{\theta}_{i}\left(\int_{0}^{\min \left\{t, a_{1}\right\}} \int_{\hat{a}}^{s_{1}}\left\|T_{i}(\hat{a}) b_{i}\left(\phi^{i}\right)(\hat{s}-\hat{a}, t-\hat{a})\right\|_{1} \mathrm{~d} \hat{s} \mathrm{~d} \hat{a}\right. \\
& \left.+\Lambda \int_{t}^{a_{1}} \int_{t}^{s_{1}}\left\|T_{i}(t) \phi_{i}(\hat{a}-t, \hat{s}-t)\right\|_{1} \mathrm{~d} \hat{s} \mathrm{~d} \hat{a}\right) \\
& +\bar{\theta}_{i-1} \frac{\psi_{i-1}}{1-\psi_{i-1}}\left(\int_{0}^{\min \left\{t, a_{1}\right\}} \int_{\hat{a}}^{s_{1}}\left\|T_{i-1}(\hat{a}) b_{i-1}\left(\phi^{i-1}\right)(\hat{s}-\hat{a}, t-\hat{a})\right\|_{1} \mathrm{~d} \hat{s} \mathrm{~d} \hat{a}\right. \\
& \left.\quad+\Lambda \int_{t}^{a_{1}} \int_{t}^{s_{1}} T_{i-1}(t) \phi_{i-1}(\hat{a}-t, \hat{s}-t) \|_{1} \mathrm{~d} \hat{s} \mathrm{~d} \hat{a}\right) \\
\leq & \bar{\theta}_{i}\left(\int_{0}^{t} e^{-\omega \hat{a}} g_{i}(t-\hat{a}) \mathrm{d} \hat{a}+\left\|\phi_{i}\right\|_{X} e^{-\omega t}\right) \\
& +\bar{\theta}_{i-1} \frac{\psi_{i-1}}{1-\psi_{i-1}}\left(\int_{0}^{t} e^{-\omega \hat{a}} g_{i-1}(t-\hat{a}) \mathrm{d} \hat{a}+\left\|\phi_{i-1}\right\|_{X} e^{-\omega t}\right) .
\end{aligned}
$$

So that

$$
\begin{aligned}
e^{\omega t} g_{i}(t) \leq & \bar{\theta}_{i}\left(\int_{0}^{t} e^{\omega \hat{a}} g_{i}(\hat{a}) \mathrm{d} \hat{a}+\left\|\phi_{i}\right\|_{X}\right) \\
& +\bar{\theta}_{i-1} \frac{\psi_{i-1}}{1-\psi_{i-1}}\left(\int_{0}^{t} e^{\omega \hat{a}} g_{i-1}(\hat{a}) \mathrm{d} \hat{a}+\left\|\phi_{i-1}\right\|_{X}\right)
\end{aligned}
$$


Hence by Gronwall's inequality

$$
\begin{gathered}
e^{\omega t} g_{i}(t) \leq \bar{\theta}_{i}\left\|\phi_{i}\right\|_{X}+\bar{\theta}_{i-1} \frac{\psi_{i-1}}{1-\psi_{i-1}}\left(\int_{0}^{t} e^{\omega \hat{a}} g_{i-1}(\hat{a}) \mathrm{d} \hat{a}+\left\|\phi_{i-1}\right\|_{X}\right) \\
+\int_{0}^{t} \bar{\theta}_{i} e^{\bar{\theta}_{i}(t-\hat{\tau})}\left(\bar{\theta}_{i}\left\|\phi_{i}\right\|_{X}+\bar{\theta}_{i-1} \frac{\psi_{i-1}}{1-\psi_{i-1}}\left(\int_{0}^{\hat{\tau}} e^{\omega \hat{a}} g_{i-1}(\hat{a}) \mathrm{d} \hat{a}+\left\|\phi_{i-1}\right\|_{X}\right)\right) \mathrm{d} \hat{\tau} \\
=\bar{\theta}_{i}\left\|\phi_{i}\right\|_{X} e^{\bar{\theta}_{i} t}+\bar{\theta}_{i-1} \frac{\psi_{i-1}}{1-\psi_{i-1}}\left(\int_{0}^{t} e^{\omega \hat{a}} g_{i-1}(\hat{a}) \mathrm{d} \hat{a}+\left\|\phi_{i-1}\right\|_{X}\right. \\
\left.+\int_{0}^{t} \bar{\theta}_{i} e^{\bar{\theta}_{i}(t-\hat{\tau})}\left(\int_{0}^{\hat{\tau}} e^{\omega \hat{a}} g_{i-1}(\hat{a}) \mathrm{d} \hat{a}+\left\|\phi_{i-1}\right\|_{X}\right) \mathrm{d} \hat{\tau}\right) .
\end{gathered}
$$

So the result is true for $i=0$.

We now assume that (3.19) is true for $i$. Then

$$
\int_{0}^{t} e^{\omega \hat{a}} g_{i}(\hat{a}) \mathrm{d} \hat{a}+\left\|\phi_{i}\right\|_{X} \leq c_{i}\left(\phi^{i}\right)\left(e^{\bar{\theta}_{i} t}-1\right)+\left\|\phi_{i}\right\|_{X} \leq c_{i}\left(\phi^{i}\right) e^{\bar{\theta}_{i} t} .
$$

Note also that

$$
e^{\bar{\theta}_{i} t}+\int_{0}^{t} \bar{\theta}_{i+1} e^{\bar{\theta}_{i+1}(t-\hat{\tau})} e^{\bar{\theta}_{i} \hat{\tau}} \mathrm{d} \hat{\tau} \leq \frac{\bar{\theta}_{i+1}}{\bar{\theta}_{i+1}-\bar{\theta}_{i}} e^{\bar{\theta}_{i+1} t}
$$

So

$$
\begin{aligned}
e^{\omega t} g_{i+1}(t) \leq & \bar{\theta}_{i+1}\left\|\phi_{i+1}\right\|_{X} e^{\bar{\theta}_{i+1} t}+\frac{\psi_{i}}{1-\psi_{i}} \bar{\theta}_{i}\left\{e^{\bar{\theta}_{i} t} c_{i}\left(\phi^{i}\right)\right. \\
& \left.+\int_{0}^{t} \bar{\theta}_{i+1} e^{\bar{\theta}_{i+1}(t-\hat{\tau})} e^{\bar{\theta}_{i} \hat{\tau}} c_{i}\left(\phi^{i}\right) \mathrm{d} \hat{\tau}\right\} \\
\leq & \bar{\theta}_{i+1} e^{\bar{\theta}_{i+1} t}\left\{\left\|\phi_{i+1}\right\|_{X}+\frac{\bar{\theta}_{i} \psi_{i}}{\left(1-\psi_{i}\right)\left(\bar{\theta}_{i+1}-\bar{\theta}_{i}\right)} c_{i}\left(\phi^{i}\right)\right\} \\
= & \bar{\theta}_{i+1} e^{\bar{\theta}_{i+1} t} c_{i+1}\left(\phi^{i+1}\right),
\end{aligned}
$$

as required. (3.15) and (3.16) now follow from the definition of $S(t)$. Now

$$
\begin{aligned}
\left\|A_{i}^{\alpha} S_{i}(t) \phi^{i}\right\|_{X} & =\int_{0}^{\min \left\{t, a_{1}\right\}} \int_{\hat{a}}^{s_{1}}\left\|A_{i}^{\alpha} T_{i}(\hat{a}) b_{i}\left(\phi^{i}\right)(\hat{s}-\hat{a}, t-\hat{a})\right\|_{1} \mathrm{~d} \hat{s} \mathrm{~d} \hat{a} \\
& +\Lambda \int_{t}^{a_{1}} \int_{t}^{s_{1}}\left\|A_{i}^{\alpha} T_{i}(t) \phi_{i}(\hat{a}-t, \hat{s}-t)\right\|_{1} \mathrm{~d} \hat{s} \mathrm{~d} \hat{a} \\
& \leq \int_{0}^{\min \left\{t, a_{1}\right\}} M_{\alpha} \hat{a}^{-\alpha} e^{-\omega \hat{a}} \bar{\theta}_{i} c_{i}\left(\phi^{i}\right) e^{\left(\bar{\theta}_{i}-\omega\right)(t-\hat{a})} \mathrm{d} \hat{a}+\Lambda M_{\alpha} e^{-\omega t} t^{-\alpha}\left\|\phi_{i}\right\|_{X} \\
& \leq \bar{\theta}_{i} c_{i}\left(\phi^{i}\right) k_{\alpha} e^{\left(\bar{\theta}_{i}-\omega\right) t}+\Lambda M_{\alpha} e^{-\omega t} t^{-\alpha}\left\|\phi_{i}\right\|_{X},
\end{aligned}
$$

for some $k_{\alpha}>0$, and the result follows.

We will write $\hat{\omega}=\bar{\theta}_{n}-\omega$. 


\section{Local Existence of the mild solution}

Take any $\frac{1}{2}<\alpha<1$. For $p_{i} \in X_{\alpha, i}, q_{i} \in X$, set $p=\left[p_{0}, \ldots, p_{n}\right]^{t}, q=\left[q_{0}, \ldots, q_{n}\right]^{t}$. Put $v_{i}=A_{i}^{\alpha} p_{i}$, $v=A^{\alpha} p$, and $V_{i}=\int_{0}^{a_{1}} \int_{0}^{s_{1}} v_{i}(a, s) \mathrm{d} s \mathrm{~d} a$. For $f_{1} \in D\left(A_{f}\right)$ define

$$
G_{i}\left(f_{1}, v_{i}\right)=\chi_{i} \nabla \cdot\left(A_{i}^{-\alpha} v_{i} \nabla f_{1}\right):=\chi_{i} \sum_{k=1}^{3}\left(B_{k} A_{i}^{-\alpha} v_{i} \frac{\partial}{\partial x_{k}} f_{1}+A_{i}^{-\alpha} v_{i} \frac{\partial^{2}}{\partial x_{k}^{2}} f_{1}\right),
$$

and set $G^{i}\left(f_{1}, v^{i}\right)=\left[G_{0}\left(f_{1}, v_{0}\right), \cdots, G_{i}\left(f_{1}, v_{i}\right)\right]^{t}$ and $G\left(f_{1}, v\right):=G^{n}\left(f_{1}, v^{n}\right)$.

In order to write the problem more efficiently set

$$
H_{i}\left(x, a, s, w, v_{i}, q_{i}\right)=-\left(\mu_{i}(x, a, s, w, N)+\sigma_{i}(x, a, s, w, N)\right) A_{i}^{-\alpha} v_{i}+\tau_{i}(x, a, s, w, N) q_{i},
$$

with $H^{i}\left(x, a, s, w, v^{i}, q^{i}\right)=\left[H_{0}\left(x, a, s, w, v_{0}, q_{0}\right), \cdots, H_{i}\left(x, a, s, w, v_{i}, q_{i}\right)\right]^{t}$ and $H(x, a, s, w, v, q):=H^{n}\left(x, a, s, w, v^{n}, q^{n}\right)$. Also let $E(x, a, s, w, v, q)(t, \sigma)$ be the matrix with $\exp -\int_{\sigma}^{t}\left(\nu_{i}(x, a, s, w(\hat{\tau}), N(\hat{\tau}))+\tau_{i}(x, a, s, w(\hat{\tau}), N(\hat{\tau}))\right) \mathrm{d} \hat{\tau}$ on the diagonal and zero elsewhere, and $\sigma(x, a, s, w, N)$ be the matrix with $\sigma_{i}(x, a, s, w, N)$ on the diagonal and zero elsewhere. Here $N$ is given in terms of $v$ and $q$ by $N=\sum_{i=0}^{n} A_{i}^{-\alpha} V_{i}+Q$.

In the following, where appropriate, we suppress the variables $x, a$ and $s$ in $\lambda_{f}(x, m, w, P)$, $\rho_{m}(x, P), \mu_{i}(x, a, s, w, N), H_{i}\left(x, a, s, w, v_{i}, q_{i}\right)$ etc. We consider the mild form of equations (2.1)(2.10):

$$
\begin{aligned}
f(t)= & T_{f}(t) f_{0}-\int_{0}^{t} T_{f}(t-\hat{\tau}) \lambda_{f}(m(\hat{\tau}), w(\hat{\tau}), P(\hat{\tau})) f(\hat{\tau}) \mathrm{d} \hat{\tau} \\
m(t)= & T_{m}(t) m_{0}+\int_{0}^{t} T_{m}(t-\hat{\tau}) \rho_{m}(P(\hat{\tau})) P(\hat{\tau}) \mathrm{d} \hat{\tau} \\
w(t)= & T_{w}(t) w_{0}+\int_{0}^{t} T_{w}(t-\hat{\tau})\left(\beta_{w}(f(\hat{\tau})) f(\hat{\tau})\right. \\
& \left.-\mu_{w}(w(\hat{\tau}), P(\hat{\tau}), Q(\hat{\tau})) w(\hat{\tau})\right) \mathrm{d} \hat{\tau} \\
p(t)= & S(t) \phi+\int_{0}^{t} S(t-\hat{\tau})\left(-G\left(f(\hat{\tau}), A^{\alpha} p(\hat{\tau})\right)+H\left(w(\hat{\tau}), A^{\alpha} p(\hat{\tau}), q(\hat{\tau})\right)\right) \mathrm{d} \hat{\tau} \\
q_{i}(t)= & \exp -\int_{0}^{t}\left(\nu_{i}(w(\hat{\tau}), N(\hat{\tau}))+\tau_{i}(w(\hat{\tau}), N(\hat{\tau}))\right) \mathrm{d} \hat{\tau} \zeta_{i} \\
+ & \int_{0}^{t} \exp -\int_{\hat{\sigma}}^{t}\left(\nu_{i}(w(\hat{\tau}), N(\hat{\tau}))+\tau_{i}(w(\hat{\tau}), N(\hat{\tau}))\right) \mathrm{d} \hat{\tau} \sigma_{i}(w(\hat{\sigma}), N(\hat{\sigma})) p_{i}(\hat{\sigma}) \mathrm{d} \hat{\sigma}
\end{aligned}
$$

So, if $\phi \in X_{\alpha}, v(t)=A^{\alpha} p(t)$ satisfies

$$
v(t)=S(t) A^{\alpha} \phi+\int_{0}^{t} A^{\alpha} S(t-\hat{\tau})(-G(f(\hat{\tau}), v(\hat{\tau}))+H(w(\hat{\tau}), v(\hat{\tau}), q(\hat{\tau}))) \mathrm{d} \hat{\tau} .
$$


As in [6] we now consider a fixed point problem in $Y=C\left(\left[0, t_{0}\right] ; Z\right)$, for suitable $t_{0}$. For $\phi \in X_{\alpha}$ and $\zeta=\left[\zeta_{0}, \ldots, \zeta_{n}\right]^{t} \in X$ set $y_{0}=\left[f_{0}, m_{0}, w_{0}, A^{\alpha} \phi, \zeta\right]^{t}$. Suppose $y_{0} \in Z$ and $\left\|y_{0}\right\|_{Y}=R$ and let $N_{R}=\left\{y \in Y:\|y\|_{Y} \leq\left(M_{f}+9\right) R\right\}$. For $y=[f, m, w, v, q]^{t} \in Y$, we define $\mathcal{F}$ by

$$
\mathcal{F} y(t)=\left[\begin{array}{l}
T_{f}(t) f_{0}-\int_{0}^{t} T_{f}(t-\hat{\tau}) \lambda_{f}(m(\hat{\tau}), w(\hat{\tau}), P(\hat{\tau})) f(\hat{\tau}) \mathrm{d} \hat{\tau} \\
T_{m}(t) m_{0}+\int_{0}^{t} T_{m}(t-\hat{\tau}) \rho_{m}(P(\hat{\tau})) P(\hat{\tau}) \mathrm{d} \hat{\tau} \\
T_{w}(t) w_{0}+\int_{0}^{t} T_{w}(t-\hat{\tau})\left(\beta_{w}(f(\hat{\tau})) f(\hat{\tau})-\mu_{w}(w(\hat{\tau}), P(\hat{\tau}), Q(\hat{\tau})) w(\hat{\tau})\right) \mathrm{d} \hat{\tau} \\
S(t) A^{\alpha} \phi+\int_{0}^{t} A^{\alpha} S(t-\hat{\tau})(-G(f(\hat{\tau}), v(\hat{\tau}))+H(w(\hat{\tau}), v(\hat{\tau}), q(\hat{\tau}))) \mathrm{d} \hat{\tau} \\
E(w, v, q)(t, 0) \zeta+\int_{0}^{t} E(w, v, q)(t, \hat{\tau}) \sigma(w(\hat{\tau}), N(\hat{\tau})) A^{-\alpha} v(\hat{\tau}) \mathrm{d} \hat{\tau}
\end{array}\right],
$$

where $P(t)=\sum_{i=0}^{n} A_{i}^{-\alpha} V_{i}(t)$.

We make the following assumptions on the data of the problem.

$\mathrm{H}(4 . .1) \quad \lambda_{f}: \Omega \times L^{1}(\Omega) \times L^{1}(\Omega) \times L^{1}(\Omega) \rightarrow \mathbb{R}, \lambda_{f}(\cdot, m, w, P) \in C^{2}(\bar{\Omega})$ with $\left.B \lambda_{f}(\cdot, m, w, P)\right|_{\partial \Omega}$ $=0$ for all $m, w, P \in L^{1}(\Omega)$. In addition, for any $R>0$, there is a constant $H_{R}>0$, independent of $x$, such that if $\max \left\{\left\|m_{j}\right\|_{1},\left\|w_{j}\right\|_{1},\left\|P_{j}\right\|_{1}\right\} \leq R, j=1,2$, then for all $x \in \Omega$,

$$
\begin{aligned}
& \max \left\{\left|\lambda_{f}\left(x, m_{1}, w_{1}, P_{1}\right)-\lambda_{f}\left(x, m_{2}, w_{2}, P_{2}\right)\right|,\right. \\
& \sum_{k=1}^{3}\left|\frac{\partial}{\partial x_{k}} \lambda_{f}\left(x, m_{1}, w_{1}, P_{1}\right)-\frac{\partial}{\partial x_{k}} \lambda_{f}\left(x, m_{2}, w_{2}, P_{2}\right)\right|, \\
& \left.\sum_{k=1}^{3}\left|\frac{\partial^{2}}{\partial x_{k}^{2}} \lambda_{f}\left(x, m_{1}, w_{1}, P_{1}\right)-\frac{\partial^{2}}{\partial x_{k}^{2}} \lambda_{f}\left(x, m_{2}, w_{2}, P_{2}\right)\right|\right\} \\
& \leq H_{R}\left\{\left\|m_{1}-m_{2}\right\|_{1}+\left\|w_{1}-w_{2}\right\|_{1}+\left\|P_{1}-P_{2}\right\|_{1}\right\} .
\end{aligned}
$$

$\mathrm{H}(4 . .2) \quad \rho_{m}: \Omega \times L^{1}(\Omega) \rightarrow \mathbb{R}, \mu_{w}: \Omega \times L^{1}(\Omega) \times L^{1}(\Omega) \times L^{1}(\Omega) \rightarrow \mathbb{R}, \beta_{w}: \Omega \times C(\Omega) \rightarrow \mathbb{R}$ are all measurable in $x$ and locally Lipschitz continuous, uniformly with respect to $x$, and $\mu_{i}, \sigma_{i}, \tau_{i}, \nu_{i}$ : $\Omega \times\left(0, a_{1}\right) \times\left(0, s_{1}\right) \times L^{1}(\Omega) \times L^{1}(\Omega) \rightarrow \mathbb{R}$, are all measurable in $x, a, s$ and locally Lipschitz continuous, uniformly with respect to $x, a, s$, i.e. for any $R>0$, there is a constant $J_{R}>0$, independent of $x, a, s$, such that, if $\max \left\{\left\|w_{j}\right\|_{1},\left\|P_{j}\right\|_{1},\left\|f_{j}\right\|_{\infty}\right\} \leq R, j=1,2$, then for all $x \in \Omega$, $(a, s) \in\left(0, a_{1}\right) \times\left(0, s_{1}\right)$,

$$
\begin{aligned}
\left|\rho_{m}\left(x, P_{1}\right)-\rho_{m}\left(x, P_{2}\right)\right| & \leq J_{R}|| P_{1}-P_{2} \|_{1} \\
\left|\mu_{w}\left(x, w_{1}, P_{1}, Q_{1}\right)-\mu_{w}\left(x, w_{2}, P_{2}, Q_{2}\right)\right| & \leq J_{R}\left\{|| w_{1}-w_{2}\left\|_{1}+\right\| P_{1}-P_{2}\left\|_{1}+\right\| Q_{1}-Q_{2} \|_{1}\right\}, \\
\left|\xi\left(x, a, s, w_{1}, N_{1}\right)-\xi\left(x, a, s, w_{2}, N_{2}\right)\right| & \leq J_{R}\left\{|| w_{1}-w_{2}\left\|_{1}+\right\| N_{1}-N_{2} \|_{1}\right\}, \\
\left|\beta_{w}\left(x, f_{1}\right)-\beta_{w}\left(x, f_{2}\right)\right| & \leq J_{R}|| f_{1}-f_{2} \|_{\infty},
\end{aligned}
$$

where $\xi$ represents any of $\mu_{i}, \sigma_{i}, \tau_{i}, \nu_{i}$. Also $\rho_{m}(\cdot, 0), \mu_{w}(\cdot, 0,0,0), \beta_{w}(\cdot, 0) \in L^{\infty}(\Omega)$ and

$$
\xi(\cdot, \cdot, \cdot, 0,0) \in L^{\infty}\left(\Omega \times\left(0, a_{1}\right) \times\left(0, s_{1}\right)\right) .
$$

Condition $\mathrm{H}(4 . .1)$ implies regularity of $f(t)$ necessary to deal with the haptotaxis term $G(f(t), v(t))$. These properties of $f(t)$ are proved in Lemma 3 of [6]. In Lemma 4 of [6] we see how they apply to the study of $G(f(t), v(t))$. That is we have 
Lemma 2. (i) For $f_{1} \in D\left(A_{f}\right)$ and $V_{1} \in L^{1}(\Omega), \frac{1}{2}<\alpha<1$, define

$$
G_{i}\left(f_{1}, V_{1}\right)=\chi_{i} \nabla \cdot\left(A_{i}^{-\alpha} V_{1} \nabla f_{1}\right):=\chi_{i} \sum_{k=1}^{3}\left(B_{k} A_{i}^{-\alpha} V_{1} \frac{\partial}{\partial x_{k}} f_{1}+A_{i}^{-\alpha} V_{1} \frac{\partial^{2}}{\partial x_{k}^{2}} f_{1}\right) .
$$

Then there exists a constant $L_{\alpha}>0$ such that

$$
\left\|G_{i}\left(f_{1}, V_{1}\right)\right\|_{1} \leq L_{\alpha}\left\|V_{1}\right\|_{1}\left\|A_{f} f_{1}\right\|_{\infty} .
$$

(ii) Also for $v_{1} \in X$,

$$
\left\|G_{i}\left(f_{1}, v_{1}\right)\right\|_{X} \leq L_{\alpha}\left\|v_{1}\right\|_{X}\left\|A_{f} f_{1}\right\|_{\infty} .
$$

(iii) So in particular if $f_{1} \in D\left(A_{f}\right)$ and $h(a, s)$ is a function in $X$ continuous at $\left(a_{0}, s_{0}\right)$ then also $G_{i}\left(f_{1}, h(a, s)\right)$ belongs to $X$ and is continuous at $\left(a_{0}, s_{0}\right)$.

(iv) For $y=[f, m, w, v, q]^{t} \in Y$, then $G(f(\cdot), V(\cdot)) \in C\left(\left[0, t_{0}\right] ; L^{1}(\Omega)^{n+1}\right)$ and $G(f(\cdot), v(\cdot)) \in$ $C\left(\left[0, t_{0}\right] ; X^{n+1}\right)$.

Note that $\left\|V_{i}(t)\right\|_{1} \leq\left\|v_{i}(t)\right\|_{X}$ and

$$
\|N(t)\|_{1} \leq \sum_{i=0}^{n}\left\|A_{i}^{-\alpha} v_{i}(t)\right\|_{X}+\sum_{i=0}^{n}\left\|q_{i}(t)\right\|_{X} \leq H\|v(t)\|_{X^{n+1}}+\|q(t)\|_{X^{n+1}} \leq H\|y\|_{Y} .
$$

Suppose now that $y, \tilde{y} \in N_{R}$. So from $\mathrm{H}(4 . .2)$ we have

$$
\begin{aligned}
\left|\mu_{i}(x, a, s, w(\tau), N(\tau))\right| \leq & \left|\mu_{i}(x, a, s, w(\tau), N(\tau))-\mu_{i}(x, a, s, 0,0)\right|+\left|\mu_{i}(x, a, s, 0,0)\right| \\
& \leq J_{\left(M_{f}+9\right) R H}\left(\|w(\tau)\|_{1}+\|N(\tau)\|_{1}\right)+\left\|\mu_{i}(0,0)\right\|_{\infty} \\
& \leq J_{\left(M_{f}+9\right) R H}\left(M_{f}+9\right) R H+\left\|\mu_{i}(0,0)\right\|_{\infty}=D_{i}, \text { say }
\end{aligned}
$$

and hence

$$
\| \mu_{i}\left(x, a, s, w(\tau), N(\tau) \|_{\infty} \leq D_{i}\right.
$$

Similarly

$$
\begin{array}{r}
\left|\mu_{i}(x, a, s, w(\tau), N(\tau))-\mu_{i}(x, a, s, \tilde{w}(\tau), \tilde{N}(\tau))\right| \leq J_{\left(M_{f}+9\right) R H}\left(\|w(\tau)-\tilde{w}(\tau)\|_{1}\right. \\
\left.+\|N(\tau)-\tilde{N}(\tau)\|_{1}\right) \leq J_{\left(M_{f}+9\right) R H} H\|y-\tilde{y}\|_{Y},
\end{array}
$$

hence

$$
\left\|\mu_{i}(x, a, s, w(\tau), N(\tau))-\mu_{i}(x, a, s, \tilde{w}(\tau), \tilde{N}(\tau))\right\|_{\infty} \leq J_{\left(M_{f}+9\right) R H} H\|y-\tilde{y}\|_{Y} .
$$

Similar inequalities hold for $\nu_{i}, \sigma_{i}$ and $\tau_{i}$.

Hence adapting the proofs of Lemmas 5,6 and 7 of [6] it is easy to see that $\mathcal{F}: Y \rightarrow Y$ is well defined, that for $t_{0}>0$ sufficiently small, $\mathcal{F}\left(N_{R}\right) \subset N_{R}$ and $\mathcal{F}$ is a contraction on $N_{R}$.

As a consequence we have the the following local existence and uniqueness result.

Theorem 3. Suppose that hypotheses H(3..1), H(4..1) and H(4..2) hold. Let $\frac{1}{2}<\alpha<1$ and take $\left[f_{0}, m_{0}, w_{0}, \phi, \zeta\right]^{t} \in D\left(A_{f}\right) \times L^{1}(\Omega) \times L^{1}(\Omega) \times X_{\alpha} \times X^{n+1}$. Then, for $t_{0}>0$ small enough, the problem (4.1)-(4.5) has a unique mild solution $[f, m, w, p, q]^{t} \in C\left(\left[0, t_{0}\right] ; D\left(A_{f}\right) \times L^{1}(\Omega) \times\right.$ $\left.L^{1}(\Omega) \times X_{\alpha} \times X^{n+1}\right)$. 


\section{Uniqueness, continuous dependence on the initial data, glo- bal existence}

We now make the additional assumption

$\mathrm{H}(5 . .1) \lambda_{f}, \frac{\partial}{\partial x_{j}} \lambda_{f}, \frac{\partial^{2}}{\partial x_{j}^{2}} \lambda_{f}(j=1,2,3), \rho_{m}, \beta_{w}, \mu_{w}, \mu_{i}, \nu_{i}, \sigma_{i}$ and $\tau_{i}, i=0, \ldots, n$, are all globally bounded.

Then a natural adaptation of the proof of Proposition 1 of [6] shows that the solutions of (4.1)(4.5) depend continuously on the initial data and are unique. That is, if $U_{\alpha}=C\left(\left[0, t_{0}\right] ; D\left(A_{f}\right) \times\right.$ $\left.L^{1}(\Omega) \times L^{1}(\Omega) \times X_{\alpha} \times X^{n+1}\right)=C\left(\left[0, t_{0}\right] ; Z_{\alpha}\right)$, then, given any $\hat{u}_{0} \in Z_{\alpha}$ and $\epsilon>0$ there exists $\delta>0$ such that $\left\|u_{0}-\hat{u}_{0}\right\|_{Z_{\alpha}}<\delta$ implies $\|u-\hat{u}\|_{U_{\alpha}}<\epsilon$. Here $u$ and $\hat{u}$ are the solutions with initial data respectively $u_{0}$ and $\hat{u}_{0}$. The global existence of solutions follows easily.

Theorem 4. Suppose that the conditions of Theorem 3 hold and also hypothesis H(5..1). Then the problem (4.1)-(4.5) has a unique mild global solution.

The proof is similar to the proof of Theorem 2 of [6].

\section{Characteristics and regularity when there is no quiescence}

Our goal now is to consider regularity, positivity, and the existence of growth bounds for our problem.

If we take initial data $\left[f_{0}, m_{0}, w_{0}, \phi, \zeta\right]^{t} \in Z_{\alpha}, \frac{1}{2}<\alpha<1$, then we know from Theorem 4 that there is a unique mild global solution $[f, m, w, p, q]^{t} \in C\left([0, \infty) ; Z_{\alpha}\right)$ of equations (2.1)-(2.10); so we can regard the coefficients $\lambda_{f}, \rho_{m}, \beta_{w}, \mu_{w}, \mu_{i}, \sigma_{i}, \tau_{i}$ as known functions of $x, a, s$, and $t$.

In this problem positivity is closely linked to regularity so we look first at regularity. To do this we would like to consider $p(a, s, t)$ and $q(a, s, t)$ along the characteristics $(t+c, t+d, t)$. This has two advantages. It reduces our problem to one in $L^{1}(\Omega)$ so that we can then exploit the fact that $T(t)$ is an analytic semigroup. Also, it effectively decouples the equations with respect to $i$. Now to consider $p$ along the characteristics we require conditions that force $p$ to be continuous in $(a, s)$ and this only seems possible if we have no quiescent cells. Thus in this section we consider the problem without quiescence and assume:

$\mathrm{H}(6 . .1) \phi \in C\left(\left[0, a_{1}\right] \times\left[0, s_{1}\right] ; D\left(A^{\alpha}\right)\right), \frac{1}{2}<\alpha<1$.

$\mathrm{H}(6 . .2)$ Set $\hat{\mu}_{i}=\mu_{i}+\sigma_{i}$. Then there exists a $F(w, N)>0$ which is bounded on bounded subsets of $L^{1}(\Omega)^{2}$, and $0<\vartheta \leq 1$ such that for $a, \tilde{a} \in\left[0, a_{1}\right]$ and $s, \tilde{s} \in\left[0, s_{1}\right]$

$$
\| \hat{\mu}_{i}(a, s, w, N)-\left.\hat{\mu}_{i}(\tilde{a}, \tilde{s}, w, N)\right|_{L^{\infty}(\Omega)} \leq F(w, N)\left(|a-\tilde{a}|^{\vartheta}+|s-\tilde{s}|^{\vartheta}\right) .
$$

Set $K_{i}\left(a, s, f, v_{i}\right)=-\chi_{i} \nabla \cdot\left(A_{i}^{-\alpha} v_{i} \nabla f\right)-\mu_{i}(a, s, w, N) A_{i}^{-\alpha} v_{i}-\sigma_{i}(a, s, w, N) A_{i}^{-\alpha} v_{i}$, and $K^{i}\left(a, s, f, v^{i}\right)=\left[K_{0}\left(a, s, f, v_{0}\right), \cdots, K_{i}\left(a, s, f, v_{i}\right)\right]^{t}$. Write $K(a, s, f, v):=K^{n}\left(a, s, f, v^{n}\right)$. 
We want to consider the restriction of $p(a, s, t)$ along the characteristics $(t+c, t+d, t)$, in the sets

$$
\begin{aligned}
& A_{1}=\left\{(a, s, t) \in\left[0, a_{1}\right] \times\left[0, s_{1}\right] \times[0, \infty): 0 \leq t \leq a \leq a_{1}, 0 \leq t \leq s \leq s_{1}\right\}, \\
& A_{2}=\left\{(a, s, t) \in\left[0, a_{1}\right] \times\left[0, s_{1}\right] \times[0, \infty): t>0,0 \leq a \leq \min \left\{t, a_{1}\right\}, 0 \leq a \leq s \leq s_{1}\right\}, \\
& A_{3}=\left\{(a, s, t) \in\left[0, a_{1}\right] \times\left[0, s_{1}\right] \times[0, \infty): t>0,0 \leq s \leq \min \left\{t, s_{1}\right\}, 0 \leq s \leq a \leq a_{1}\right\} .
\end{aligned}
$$

Now for $t \geq 0$ we have for a.a. $(a, s)$,

$$
p_{i}(t)(a, s)=\left(S_{i}(t) \phi^{i}\right)(a, s)+\int_{0}^{t}\left(S_{i}(t-\hat{\tau})\left(K^{i}\left(f(\hat{\tau}),\left(A^{\alpha} p\right)^{i}(\hat{\tau})\right)\right)(a, s) \mathrm{d} \hat{\tau} .\right.
$$

Suppose for motivation that, for each $t, A^{\alpha} p(t)(a, s)$ were a continuous function of $(a, s)$ so that $K(a, s, f(t), v(t)(a, s))$ is continuous in $(a, s)$. So if $0<t<a \leq a_{1}, 0<t<s \leq s_{1}$,

$$
\begin{aligned}
& p_{i}(t)(a, s)=T_{i}(t) \phi_{i}(a-t, s-t)+ \\
& \quad \int_{0}^{t} T_{i}(t-\hat{\tau})\left(K_{i}\left(a-t+\hat{\tau}, s-t+\hat{\tau}, f(\hat{\tau}), A_{i}^{\alpha} p_{i}(a-t+\hat{\tau}, s-t+\hat{\tau}, \hat{\tau})\right) \mathrm{d} \hat{\tau} .\right.
\end{aligned}
$$

If $0 \leq a<\min \left\{t, a_{1}\right\}, 0 \leq a<s \leq s_{1}$

$$
\begin{aligned}
p_{i}(t)(a, s)= & \left(S_{i}(t) \phi^{i}\right)(a, s)+\int_{0}^{t-a}\left(S_{i}(t-\hat{\tau})\left(K^{i}\left(f(\hat{\tau}),\left(A^{\alpha} p\right)^{i}(\hat{\tau})\right)\right)(a, s) \mathrm{d} \hat{\tau}\right. \\
& +\int_{t-a}^{t}\left(S_{i}(t-\hat{\tau})\left(K^{i}\left(f(\hat{\tau}),\left(A^{\alpha} p\right)^{i}(\hat{\tau})\right)\right)(a, s) \mathrm{d} \hat{\tau}\right.
\end{aligned}
$$

Using (3.10), (3.12) and (6.1) it can be shown that

$$
\begin{aligned}
& \left(S_{i}(t) \phi^{i}\right)(a, s)+\int_{0}^{t-a}\left(S_{i}(t-\hat{\tau})\left(K^{i}\left(f(\hat{\tau}),\left(A^{\alpha} p\right)^{i}(\hat{\tau})\right)\right)(a, s) \mathrm{d} \hat{\tau}\right. \\
& =T_{i}(a)\left\{\int_{0}^{a_{1}} \int_{0}^{s_{1}} \theta_{i}(\hat{a}, \hat{s}, s-a) p_{i}(\hat{a}, \hat{s}, t-a) \mathrm{d} \hat{s} \mathrm{~d} \hat{a}+\right. \\
& \left.\frac{\psi_{i-1}}{1-\psi_{i-1}} \int_{0}^{a_{1}} \int_{0}^{s_{1}} \theta_{i-1}(\hat{a}, \hat{s}, s-a) p_{i-1}(\hat{a}, \hat{s}, t-a) \mathrm{d} \hat{s} \mathrm{~d} \hat{a}\right\},
\end{aligned}
$$

which is continuous in $(a, s)$. So that

$$
\begin{aligned}
& p_{i}(t)(a, s)=T_{i}(a)\left\{\int_{0}^{a_{1}} \int_{0}^{s_{1}} \theta_{i}(\hat{a}, \hat{s}, s-a) p_{i}(\hat{a}, \hat{s}, t-a) \mathrm{d} \hat{s} \mathrm{~d} \hat{a}+\right. \\
& \left.\quad \frac{\psi_{i-1}}{1-\psi_{i-1}} \int_{0}^{a_{1}} \int_{0}^{s_{1}} \theta_{i-1}(\hat{a}, \hat{s}, s-a) p_{i-1}(\hat{a}, \hat{s}, t-a) \mathrm{d} \hat{s} \mathrm{~d} \hat{a}\right\} \\
& +\int_{t-a}^{t} T_{i}(t-\hat{\tau})\left(K_{i}\left(a-t+\hat{\tau}, s-t+\hat{\tau}, f(\hat{\tau}), A_{i}^{\alpha} p_{i}(a-t+\hat{\tau}, s-t+\hat{\tau}, \hat{\tau})\right) \mathrm{d} \hat{\tau} .(6\right.
\end{aligned}
$$


Finally if $0 \leq s<\min \left\{t, s_{1}\right\}, 0 \leq s<a \leq a_{1}$,

$$
\begin{aligned}
& p_{i}(t)(a, s)=\left(S_{i}(t) \phi^{i}\right)(a, s)+\int_{0}^{t-s}\left(S_{i}(t-\hat{\tau})\left(K^{i}\left(f(\hat{\tau}),\left(A^{\alpha} p\right)^{i}(\hat{\tau})\right)\right)(a, s) \mathrm{d} \hat{\tau}\right. \\
& +\int_{t-s}^{t}\left(S_{i}(t-\hat{\tau})\left(K^{i}\left(f(\hat{\tau}),\left(A^{\alpha} p\right)^{i}(\hat{\tau})\right)\right)(a, s) \mathrm{d} \hat{\tau}\right. \\
= & \int_{t-s}^{t} T_{i}(t-\hat{\tau})\left(K_{i}\left(a-t+\hat{\tau}, s-t+\hat{\tau}, f(\hat{\tau}), A_{i}^{\alpha} p_{i}(a-t+\hat{\tau}, s-t+\hat{\tau}, \hat{\tau})\right) \mathrm{d} \hat{\tau} .\right.
\end{aligned}
$$

Motivated by the above we make the following definitions:

If $0<c \leq a_{1}, 0<d \leq s_{1}$,

$$
\tilde{p}_{i, c, d}(t)=T_{i}(t) \phi_{i}(c, d)+\int_{0}^{t} T_{i}(t-\sigma) K_{i}\left(\sigma+c, \sigma+d, f(\sigma), A_{i}^{\alpha} \tilde{p}_{i, c, d}(\sigma)\right) \mathrm{d} \sigma .
$$

If $c<0, c<d \leq s_{1}$

$$
\begin{aligned}
& \tilde{p}_{i, c, d}(t)=T_{i}(t+c)\left\{\int_{0}^{a_{1}} \int_{0}^{s_{1}} \theta_{i}(\hat{a}, \hat{s}, d-c) p_{i}(\hat{a}, \hat{s},-c) \mathrm{d} \hat{s} \mathrm{~d} \hat{a}+\right. \\
&\left.\frac{\psi_{i-1}}{1-\psi_{i-1}} \int_{0}^{a_{1}} \int_{0}^{s_{1}} \theta_{i-1}(\hat{a}, \hat{s}, d-c) p_{i-1}(\hat{a}, \hat{s},-c) \mathrm{d} \hat{s} \mathrm{~d} \hat{a}\right\} \\
& \quad+\int_{-c}^{t} T_{i}(t-\sigma) K_{i}\left(\sigma+c, \sigma+d, f(\sigma), A_{i}^{\alpha} \tilde{p}_{i, c, d}(\sigma)\right) \mathrm{d} \sigma .
\end{aligned}
$$

If $d<0, d<c \leq a_{1}$

$$
\tilde{p}_{i, c, d}(t)=\int_{-d}^{t} T_{i}(t-\sigma) K_{i}\left(\sigma+c, \sigma+d, f(\sigma), A_{i}^{\alpha} \tilde{p}_{i, c, d}(\sigma)\right) \mathrm{d} \sigma .
$$

We can write these equations as the single equation, for $t \geq t_{c, d}$,

$$
\tilde{p}_{i, c, d}(t)=T_{i}\left(t-t_{c, d}\right) \tilde{p}_{i, c, d}\left(t_{c, d}\right)+\int_{t_{c, d}}^{t} T_{i}(t-\sigma) K_{i}\left(\sigma+c, \sigma+d, f(\sigma), A_{i}^{\alpha} \tilde{p}_{i, c, d}(\sigma)\right) \mathrm{d} \sigma,
$$

and if $\tilde{v}_{i, c, d}=A_{i}^{\alpha} \tilde{p}_{i, c, d}$

$$
\tilde{v}_{i, c, d}(t)=T_{i}\left(t-t_{c, d}\right) A_{i}^{\alpha} \tilde{p}_{i, c, d}\left(t_{c, d}\right)+\int_{t_{c, d}}^{t} A_{i}^{\alpha} T_{i}(t-\sigma) K_{i}\left(\sigma+c, \sigma+d, f(\sigma), \tilde{v}_{i, c, d}(\sigma)\right) \mathrm{d} \sigma
$$

if we take $t_{c, d}=0$ if $0<c \leq a_{1}, 0<d \leq s_{1}, t_{c, d}=-c$ if $c<0, c<d \leq s_{1}$ and $t_{c, d}=-d$ if $d<0, d<c \leq a_{1}$.

Note that in the case $d<0, d<c \leq a_{1}$, if we operate on (6.7) with $A_{i}^{\alpha}$ and use [21] Lemma 1.1 then we see that for all $t \geq-d$

$$
\tilde{v}_{i, c, d}(t)=0, \text { so } \quad \tilde{p}_{i, c, d}(t)=0 \text {. }
$$


It is easy to see that each of the equations (6.9) has a unique solution $\tilde{v}_{i, c, d} \in C\left(\left[t_{c, d}, \infty\right)\right.$ : $\left.L^{1}(\Omega)\right)$. We write $\tilde{p}_{c, d}=\left[\tilde{p}_{0, c, d}, \ldots, \tilde{p}_{n, c, d}\right]^{t}$ etc.

We now show that $p(a, s, t)=\tilde{p}_{a-t, s-t}(t)$ and is continuous in $(a, s)$ for $s \neq t, a \neq t, a \neq s$. Define

$$
\tilde{p}(a, s, t)=\tilde{p}_{a-t, s-t}(t)
$$

and

$$
\tilde{v}(a, s, t)=A^{\alpha} \tilde{p}(a, s, t)=\tilde{v}_{a-t, s-t}(t) .
$$

Thus, if $0<t<a \leq a_{1}$ and $0<t<s \leq s_{1}, \tilde{p}_{i}(a, s, t)$ satisfies (6.2) and if $0 \leq s<\min \left\{t, s_{1}\right\}$ and $0 \leq s<a \leq a_{1}$ it satisfies (6.4). If $0 \leq a<\min \left\{t, a_{1}\right\}$ and $0 \leq a<s \leq s_{1}$,

$$
\begin{aligned}
& \tilde{p}_{i}(t)(a, s)=T_{i}(a)\left\{\int_{0}^{a_{1}} \int_{0}^{s_{1}} \theta_{i}(\hat{a}, \hat{s}, s-a) p_{i}(\hat{a}, \hat{s}, t-a) \mathrm{d} \hat{s} \mathrm{~d} \hat{a}+\right. \\
& \left.\quad \frac{\psi_{i-1}}{1-\psi_{i-1}} \int_{0}^{a_{1}} \int_{0}^{s_{1}} \theta_{i-1}(\hat{a}, \hat{s}, s-a) p_{i-1}(\hat{a}, \hat{s}, t-a) \mathrm{d} \hat{s} \mathrm{~d} \hat{a},\right\} \\
& +\int_{t-a}^{t} T_{i}(t-\hat{\tau})\left(K_{i}\left(a-t+\hat{\tau}, s-t+\hat{\tau}, f(\hat{\tau}), A_{i}^{\alpha} \tilde{p}_{i}(a-t+\hat{\tau}, s-t+\hat{\tau}, \hat{\tau})\right) \mathrm{d} \hat{\tau}(6.11)\right.
\end{aligned}
$$

If we operate on these equations with $A_{i}^{\alpha}$ we will get equivalent equations satisfied by $\tilde{v}$. As in [7] Lemma 4, we have

Lemma 5. For fixed $t \geq 0, \tilde{v}_{i, c, d}(t)$ is continuous in $(c, d)$ from $\left(-\infty, a_{1}\right] \times\left(-\infty, s_{1}\right]$ to $L^{1}(\Omega)$, except possibly along $\left\{(c, d) ; c=0,0 \leq d \leq s_{1}\right\},\left\{(c, d) ; d=0,0 \leq c \leq a_{1}\right\}$ or $\{(c, d) ; c=$ $d \leq 0\}$ where the right and left hand limits exist.

Thus it follows that for fixed $t \geq 0, \tilde{v}(t)(a, s)=\tilde{v}(a, s, t)$, and hence also $\tilde{p}(t)(a, s)=$ $\tilde{p}(a, s, t)$, is continuous from $\left[0, a_{1}\right] \times\left[0, s_{1}\right]$ to $L^{1}(\Omega)^{n+1}$ except possibly along $a=t, s=t$ or $a=s$ where the left and right hand limits exist. So $\tilde{p}(a, s, t) \in L^{1}(\Omega)^{n+1}$ for all $t \geq 0$, and $(a, s) \in\left[0, a_{1}\right] \times\left[0, s_{1}\right]$, and $\tilde{p}(t) \in L^{1}\left(\left(0, a_{1}\right) \times\left(0, s_{1}\right) ; D\left(A^{\alpha}\right)\right)$.

Proof. The proof is very similar to that in [7] Lemma 4 once we note that $\hat{\mu}_{i}(\sigma+c, \sigma+d, w(\sigma), N(\sigma))$ is continuous in $(c, d)$, uniformly with respect to $\sigma$ for $\sigma \in[0, t]$ and that if $c<0, c<d \leq s_{1}$

$$
\begin{aligned}
& T_{i}(t+c)\left\{\int_{0}^{a_{1}} \int_{0}^{s_{1}} \theta_{i}(\hat{a}, \hat{s}, d-c) A_{i}^{\alpha} p_{i}(\hat{a}, \hat{s},-c) \mathrm{d} \hat{s} \mathrm{~d} \hat{a}\right. \\
&\left.+\frac{\psi_{i-1}}{1-\psi_{i-1}} \int_{0}^{a_{1}} \int_{0}^{s_{1}} \theta_{i-1}(\hat{a}, \hat{s}, d-c) A_{i}^{\alpha} p_{i-1}(\hat{a}, \hat{s},-c) \mathrm{d} \hat{s} \mathrm{~d} \hat{a}\right\}
\end{aligned}
$$

is continuous in $(c, d)$.

Now

Lemma 6. For all $t \geq 0, \tilde{v}(t)=v(t)$, and hence also $\tilde{p}(t)=p(t)$. So for fixed $t, v(a, s, t)$, and hence also $p(a, s, t)$, is continuous from $\left[0, a_{1}\right] \times\left[0, s_{1}\right]$ to $L^{1}(\Omega)^{n+1}$ except possibly along $a=t$, $s=t$ and $a=s$, where the left and right hand limits exist. Hence $p(a, s, t)=\tilde{p}_{a-t, s-t}(t)$ and $v(a, s, t)=\tilde{v}_{a-t, s-t}(t)$. 
Proof. Note that for each $t \geq 0 K(a, s, f(t), \tilde{v}(t)(a, s))$ is continuous in $(a, s)$ except possibly along $a=t, s=t$ and $a=s$, so that $S(t-\sigma) K(f(\sigma), \tilde{v}(\sigma))$ is also a continuous function of $(a, s)$ for $a \neq \sigma, s \neq \sigma, a \neq s, a \neq t-\sigma, s \neq t-\sigma$. Now if $0<t<a \leq a_{1}, 0<t<s \leq s_{1}$,

$$
\tilde{v}(t)(a, s)=A^{\alpha}(S(t) \phi)(a, s)+\int_{0}^{t} A^{\alpha}(S(t-\hat{\tau})(K(f(\hat{\tau}), \tilde{v}(\hat{\tau})))(a, s)) \mathrm{d} \hat{\tau} .
$$

If $0 \leq a<\min \left\{t, a_{1}\right\}, 0 \leq a<s \leq s_{1}$

$$
\begin{aligned}
\tilde{v}_{i}(t)(a, s)= & A_{i}^{\alpha} T_{i}(a)\left\{\int_{0}^{a_{1}} \int_{0}^{s_{1}} \theta_{i}(\hat{a}, \hat{s}, s-a) p_{i}(\hat{a}, \hat{s}, t-a) \mathrm{d} \hat{s} \mathrm{~d} \hat{a}+\right. \\
& \left.\frac{\psi_{i-1}}{1-\psi_{i-1}} \int_{0}^{a_{1}} \int_{0}^{s_{1}} \theta_{i-1}(\hat{a}, \hat{s}, s-a) p_{i-1}(\hat{a}, \hat{s}, t-a) \mathrm{d} \hat{s} \mathrm{~d} \hat{a},\right\} \\
& +\int_{t-a}^{t} A_{i}^{\alpha}\left(S_{i}(t-\hat{\tau})\left(K^{i}\left(f(\hat{\tau}), \tilde{v}^{i}(\hat{\tau})\right)\right)(a, s)\right) \mathrm{d} \hat{\tau} .
\end{aligned}
$$

Finally if $0 \leq s<\min \left\{t, s_{1}\right\}, 0 \leq s<a \leq a_{1}$,

$$
\tilde{v}(t)(a, s)=\int_{t-s}^{t} A^{\alpha}(S(t-\hat{\tau})(K(f(\hat{\tau}), \tilde{v}(\hat{\tau})))(a, s)) \mathrm{d} \hat{\tau} .
$$

Thus if $t \geq 0$ and $\Lambda$ is as in (3.9)

$$
\begin{aligned}
\| \tilde{v}(t) & -v(t) \|_{X^{n+1}} \\
\leq & \Lambda \int_{t}^{s_{1}} \int_{t}^{a_{1}} \int_{0}^{t}\left\|A^{\alpha}(S(t-\sigma)(K(f(\sigma), \tilde{v}(\sigma)-v(\sigma)))(a, s))\right\|_{L^{1}(\Omega)^{n+1}} \mathrm{~d} \sigma \mathrm{d} s \mathrm{~d} a \\
& +\int_{0}^{\min \left\{t, a_{1}\right\}} \int_{a}^{s_{1}} \int_{t-a}^{t}\left\|A^{\alpha}(S(t-\sigma)(K(f(\sigma), \tilde{v}(\sigma)-v(\sigma)))(a, s))\right\|_{L^{1}(\Omega)^{n+1}} \mathrm{~d} \sigma \mathrm{d} s \mathrm{~d} a \\
& +\int_{0}^{\min \left\{t, s_{1}\right\}} \int_{s}^{a_{1}} \int_{t-s}^{t}\left\|A^{\alpha}(S(t-\sigma)(K(f(\sigma), \tilde{v}(\sigma)-v(\sigma)))(a, s))\right\|_{L^{1}(\Omega)^{n+1}} \mathrm{~d} \sigma \mathrm{d} s \mathrm{~d} a \\
& \leq \int_{0}^{t}\left\|A^{\alpha}(S(t-\sigma)(K(f(\sigma), \tilde{v}(\sigma)-v(\sigma))))\right\|_{X^{n+1}} \mathrm{~d} \sigma \\
& \leq \int_{0}^{t} k_{1}(t-\sigma)^{-\alpha} e^{\hat{\omega}(t-\sigma)}\|\tilde{v}(\sigma)-v(\sigma)\|_{X^{n+1}} \mathrm{~d} \sigma,
\end{aligned}
$$

where $k_{1}>0$ is constant. Thus, by [21] Lemma 1.1, $\tilde{v}(t)=v(t)$, so that $\tilde{p}(t)=p(t)$.

Note that it now follows from (6.3) that for $t>0$

$$
\begin{aligned}
p_{i}(t)(0, s)= & \int_{0}^{a_{1}} \int_{0}^{s_{1}} \theta_{i}(\hat{a}, \hat{s}, s) p_{i}(\hat{a}, \hat{s}, t) \mathrm{d} \hat{s} \mathrm{~d} \hat{a}+ \\
& \frac{\psi_{i-1}}{1-\psi_{i-1}} \int_{0}^{a_{1}} \int_{0}^{s_{1}} \theta_{i-1}(\hat{a}, \hat{s}, s) p_{i-1}(\hat{a}, \hat{s}, t) \mathrm{d} \hat{s} \mathrm{~d} \hat{a}, \text { and from (6.4) } \\
p_{i}(t)(a, 0)= & 0 .
\end{aligned}
$$


In fact, from (6.10), $p(a, s, t)=0$ in $0 \leq s<a \leq a_{1}, 0 \leq s<\min \left\{t, s_{1}\right\}$.

Assume that

$\mathrm{H}\left(6 . .3\right.$ ) (a) $\mu_{w}, \mu_{i}, \sigma_{i}$, are all independent of $P, Q$ and $N$; (b) $\rho_{m}$ is independent of $x$.

Motivated by [22] Chapter 1 we define

$$
D p(a, s, t):=\lim _{h \rightarrow 0} \frac{p(a+h, s+h, t+h)-p(a, s, t)}{h} .
$$

As in [7] Proposition 6 we can prove

Proposition 7. Suppose that hypotheses $H(3 . .1), H(4 . .1), H(4 . .2), H(5 . .1), H(6.1), H(6 . .2)$ and $H(6 . .3)(a)$ hold. Take $\left[f_{0}, m_{0}, w_{0}, \phi\right]^{t} \in Z_{\alpha, p}$ where $\frac{1}{2}<\alpha<1$. Then $\tilde{p}_{i, c, d} \in$ $C\left(\left[t_{c, d}, \infty\right) ; L^{1}(\Omega)\right) \cap C^{1}\left(\left(t_{c, d}, \infty\right) ; L^{1}(\Omega)\right)$, and for $t>t_{c, d}, \tilde{p}_{i, c, d}(t) \in D\left(A_{i}\right)$ and

$$
\begin{aligned}
\frac{\mathrm{d}}{\mathrm{d} t} \tilde{p}_{i, c, d}(t)= & \delta_{i} \Delta \tilde{p}_{i, c, d}(t)-\omega \tilde{p}_{i, c, d}(t)-\chi_{i} \nabla \cdot\left(\tilde{p}_{i, c, d}(t) \nabla f(t)\right) \\
& -\mu_{i}(t+c, t+d, w(t)) \tilde{p}_{i, c, d}(t)-\sigma_{i}(t+c, t+d, w(t)) \tilde{p}_{i, c, d}(t) .
\end{aligned}
$$

Proof. As in [7] Proposition 6, using [13] Theorem 4.3.1, $A_{f} f$ and $w$ are locally Hölder continuous, and adapting the proof of [13] Theorem 6.3.1 so is $\tilde{v}_{i, c, d}$. But now $\hat{\mu}_{i}(t+c, t+d, w(t))$ is locally Hölder continuous, so, using Lemma $2, K_{i}\left(t+c, t+d, f(t), \tilde{v}_{i, c, d}(t)\right)$ is locally Hölder continuous. The result now follows.

The following result is now proved almost exactly as in [7]

Theorem 8. Suppose that hypotheses $H(3 . .1), H(4 . .1), H(4 . .2), H(5 . .1), H(6 . .1), H(6.2)$ and $H(6 . .3)$ hold. Take $\left[f_{0}, m_{0}, w_{0}, \phi\right]^{t} \in D\left(A_{f}\right) \times L^{1}(\Omega)^{2} \times X_{\alpha}$ where $\frac{1}{2}<\alpha<1$. Then the problem (2.1)(2.4), (2.6)-(2.10), with $\tau_{i} \equiv 0$, has a unique classical solution $[f, m, w, p]^{t}$ in the sense that $f, m, w$ satisfy

$$
\begin{aligned}
& f \in C([0, \infty) ; C(\bar{\Omega})) \cap C^{1}((0, \infty) ; C(\bar{\Omega})), \text { and for } t \geq 0 f(t) \in D\left(A_{f}\right) \\
& m, w \in C\left([0, \infty) ; L^{1}(\Omega)\right) \cap C^{1}\left((0, \infty) ; L^{1}(\Omega)\right), \text { and for } t>0 m(t) \in D\left(A_{m}\right) \\
& w(t) \in D\left(A_{w}\right) ; \text { and } \\
& \frac{\mathrm{d}}{\mathrm{d} t} f(t)=\delta_{f} \Delta f(t)-\omega_{f} f(t)-\lambda_{f}(m(t), w(t), P(t)) f(t) t \geq 0, f(0)=f_{0} \\
& \frac{\mathrm{d}}{\mathrm{d} t} m(t)=\delta_{m} \Delta m(t)-\omega_{m} m(t)+\rho_{m}(P(t)) P(t) t>0, m(0)=m_{0} \\
& \frac{\mathrm{d}}{\mathrm{d} t} w(t)=\delta_{w} \Delta w(t)-\omega_{w} w(t)+\beta_{w}(f(t)) f(t)-\mu_{w}(w(t)) w(t) t>0, w(0)=w_{0}(6)
\end{aligned}
$$

$p$ satisfies (2.6) and (2.7)(for $t>0),(2.10)$, and for $t>0, p(a, s, t) \in D(A)$, and

$$
\begin{aligned}
D p_{i}(a, s, t)= & \delta_{i} \Delta p_{i}(a, s, t)-\omega p_{i}(a, s, t)-\chi_{i} \nabla \cdot\left(p_{i}(, a, s, t) \nabla f(t)\right) \\
& -\mu_{i}(a, s, w(\cdot, t)) p_{i}(a, s, t)-\sigma_{i}(a, s, w(\cdot, t)) p_{i}(a, s, t) \text { for } t>0 .
\end{aligned}
$$


Remark. If quiescence is included in the system of equations it is easy to see that if $\left[f_{0}, m_{0}, w_{0}, \phi, \zeta\right]^{t} \in Z_{\alpha}$, then it is still the case that $f, m, w$ are classical solutions of equations (2.1)-(2.3), (2.8) and (2.10). Also, in that case, as $p \in C\left([0, \infty) ; X_{\alpha}\right)$, we have $q \in C^{1}([0, \infty) ; X)$ and

$$
\frac{\mathrm{d}}{\mathrm{d} t} q_{i}(t)=-\nu_{i}(w(\cdot, t), N(\cdot, t)) q_{i}(t)+\sigma_{i}(w(\cdot, t)) p_{i}(t)-\tau_{i}(w(\cdot, t), N(\cdot, t)) q_{i}(t) .
$$

\section{Positivity when there is no quiescence}

We prove that, in the absence of quiescence, $p \geq 0$. We assume

$\mathrm{H}(7 . .1) \phi \geq 0$

We will also require

$\mathrm{H}(7 . .2)$ There exists $\epsilon>0$ such that $\theta_{i}(\hat{a}, \hat{s}, s)=0$, when $\hat{a} \in[0, \epsilon]$.

The proof is very similar to that in Section 5 of [7]. Again we show that if the initial function $\phi$ is regular enough then $\tilde{p}_{i, c, d}(t) \in C\left(\left[t_{c, d}, \infty\right) ; C(\bar{\Omega})\right)$ so that we can use ideas from [12] and [20] to prove the positivity of $\tilde{p}_{c, d}(t)$ and hence of $p(a, s, t)$. The only significant difference between our problem here and that in [7] is the study of regularity and positivity of the initial condition $\tilde{p}_{c, d}\left(t_{c, d}\right)$ for the case $c<0, c<d \leq s_{1}$. We define $A_{(r)}: D\left(A_{(r)}\right) \subset L^{1}(\Omega)^{n+1} \rightarrow L^{1}(\Omega)^{n+1}, D\left(A_{(r)}\right)=$ $\prod_{i=0}^{n} D\left(A_{i, r}\right)$, by $A_{(r)} \phi=\left[A_{0, r} \phi_{0}, \ldots, A_{n, r} \phi_{n}\right]^{t}$. We show first that $\phi \in C\left(\left[0, a_{1}\right] \times\left[0, s_{1}\right] ; D\left(A_{(r)}^{\alpha}\right)\right)$ implies that, for $-\infty<c \leq a_{1}$ and $-\infty<d \leq s_{1}, \tilde{p}_{c, d}\left(t_{c, d}\right) \in D\left(A_{(r)}^{\alpha}\right)$. Once this is done the proof of [7] carries over directly to this case except that we need to show that $\tilde{p}_{c, d}\left(t_{c, d}\right) \geq 0$.

So firstly we consider equation (6.8) as an equation in $L^{r}(\Omega)$ and suppose that $\phi_{i} \in C\left(\left[0, a_{1}\right] \times\right.$ $\left.\left[0, s_{1}\right] ; D\left(A_{i, r}^{\alpha}\right)\right)$. Exactly as in [7] it can be proved, for $-\infty<c \leq a_{1},-\infty<d \leq s_{1}$, that if

$$
\tilde{p}_{i, c, d}\left(t_{c, d}\right) \in D\left(A_{i, r}^{\alpha}\right),
$$

then $\tilde{p}_{i, c, d}(t) \in C\left(\left[t_{c, d}, \infty\right) ; D\left(A_{i, r}^{\alpha}\right)\right)$.

Thus we have only to show that $\tilde{p}_{i, c, d}\left(t_{c, d}\right) \in D\left(A_{i, r}^{\alpha}\right)$.

If $0 \leq c \leq a_{1}, 0 \leq d \leq s_{1}$ this is immediate, as $\tilde{p}_{i, c, d}(0)=\phi_{i}(c, d)$, so in this case (7.1) holds and hence $\tilde{p}_{i, c, d}(t) \in D\left(A_{i, r}^{\alpha}\right)$ for $t \geq 0$.

If $d<0$ and $d<c \leq a_{1}$ then by (6.10) $\tilde{p}_{i, c, d}(t)=0 \in D\left(A_{i, r}^{\alpha}\right)$, for $t \geq-d$.

Suppose now $-\epsilon<c<0$ and $c<d \leq s_{1}$. Then

$$
\begin{aligned}
\tilde{p}_{i, c, d}\left(t_{c, d}\right)= & \int_{\epsilon}^{a_{1}} \\
& \int_{0}^{s_{1}} \theta_{i}(\hat{a}, \hat{s}, d-c) p_{i}(\hat{a}, \hat{s},-c) \mathrm{d} \hat{s} \mathrm{~d} \hat{a}+ \\
& \frac{\psi_{i-1}}{1-\psi_{i-1}} \int_{\epsilon}^{a_{1}} \int_{0}^{s_{1}} \theta_{i-1}(\hat{a}, \hat{s}, d-c) p_{i-1}(\hat{a}, \hat{s},-c) \mathrm{d} \hat{s} \mathrm{~d} \hat{a} .
\end{aligned}
$$

But $p_{j}(\hat{a}, \hat{s},-c)=\tilde{p}_{j, \hat{a}+c, \hat{s}+c}(-c)$. Now if $\hat{a} \geq \epsilon, \hat{a}+c>0$ so if $\hat{s}+c>0, p_{j}(\hat{a}, \hat{s},-c) \in D\left(A_{j, r}^{\alpha}\right)$ from the first case above. On the other hand if $\hat{s}+c<0$ then $p_{j}(\hat{a}, \hat{s},-c)=0 \in D\left(A_{j, r}^{\alpha}\right)$ from the 
second case above. Thus, as $D\left(A_{j, r}^{\alpha}\right)$ is independent of $j,(7.1)$ again holds, so that combining the various cases, if $-\epsilon<c<0$, then

$$
\tilde{p}_{i, c, d}(t) \in D\left(A_{i, r}^{\alpha}\right) \text { for } t \geq t_{c, d} .
$$

We can now use this to show that if $-2 \epsilon<c<-\epsilon$ and $c<d \leq s_{1}$, then (7.1) holds. So again by combining the different cases we see that (7.2) holds if $-2 \epsilon<c<-\epsilon$. Continue by induction.

Now as in [7] it follows that for any $\frac{1}{2}<\beta<\alpha<1, \tilde{p}_{i, c, d}(t) \in C\left(\left[t_{c, d}, \infty\right) ; W^{2 \beta, r}(\Omega)\right)$. If we take $r>3$ then for $2 \beta>1, W^{2 \beta, r}(\Omega) \hookrightarrow C(\bar{\Omega})$ so for any $T>t_{c, d}$,

$$
\tilde{p}_{i, c, d}(t) \in C\left(\left[t_{c, d}, T\right] \times \bar{\Omega}\right) .
$$

Theorem 9. Suppose that hypotheses $H(3 . .1), H(4 . .1), H(4 . .2), H(5 . .1), H(6 . .2), H(6 . .3), H(7 . .1)$ and $H(7 . .2)$ are satisfied, that $\frac{1}{2}<\alpha<1$, and $\left[f_{0}, m_{0}, w_{0}, \phi\right]^{t} \in D\left(A_{f}\right) \times L^{1}(\Omega)^{2} \times X_{\alpha}$. Then the solutions of (4.4), with $\tau_{i} \equiv 0$, are positive.

Proof. Assume first that for some $r>3, \phi \in C\left(\left[0, a_{1}\right] \times\left[0, s_{1}\right] ; D\left(A_{(r)}^{\alpha}\right)\right)$. The proof follows that of Theorem 9 in [7]. It is easy to see from that proof that for any $-\infty<c \leq a_{1}$ and $-\infty<d \leq s_{1}$

$$
\tilde{p}_{i, c, d}\left(t_{c, d}\right) \geq 0
$$

implies that $\tilde{p}_{i, c, d}(t) \geq 0$ for $t \geq t_{c, d}$. From this the positivity of $p$ follows easily. Thus we have only to prove (7.4). But this follows from $\phi \geq 0$ using exactly the same iterative argument as above.

We now extend the result to positive $\phi \in L_{+}^{1}\left(\left(0, a_{1}\right) \times\left(0, s_{1}\right) ; D\left(A^{\alpha}\right)\right)$. We have

$$
\begin{aligned}
C_{+}\left(\left[0, a_{1}\right] \times\left[0, s_{1}\right] ; D\left(A_{(r)}^{\alpha}\right)\right) & \stackrel{d}{\hookrightarrow} L_{+}^{1}\left(\left(0, a_{1}\right) \times\left(0, s_{1}\right) ; D\left(A_{(r)}^{\alpha}\right)\right) \\
& \stackrel{d}{\hookrightarrow} L_{+}^{1}\left(\left(0, a_{1}\right) \times\left(0, s_{1}\right) ; D\left(A^{\alpha}\right)\right),
\end{aligned}
$$

where ' $\stackrel{d}{\hookrightarrow}$ ' denotes a dense continuous embedding, see [2]. Let $p(t)$ be the solution with initial data $\phi \in L_{+}^{1}\left(\left(0, a_{1}\right) \times\left(0, s_{1}\right) ; D\left(A^{\alpha}\right)\right)$. Then there exist $\phi(m) \in C_{+}\left(\left[0, a_{1}\right] \times\left[0, s_{1}\right] ; D\left(A_{(r)}^{\alpha}\right)\right)$ such that $\left\|A^{\alpha}(\phi(m)-\phi)\right\|_{X^{n+1}} \rightarrow 0$ as $m \rightarrow \infty$. Let $p(m)(t)$ be the solution with initial data $\phi(m)$. Then by continuous dependence on the initial data, for any $T>0, \sup _{t \in[0, T]} \| A^{\alpha}(p(m)(t)-$ $p(t)) \|_{X^{n+1}} \rightarrow 0$ as $m \rightarrow \infty$, and hence $\sup _{t \in[0, T]}\|p(m)(t)-p(t)\|_{X^{n+1}} \rightarrow 0$ as $m \rightarrow \infty$. Thus for any $t>0$ there exists a subsequence $p\left(m_{r}\right)(x, a, s, t) \rightarrow p(x, a, s, t)$, a.e. as $r \rightarrow \infty$ so $p \geq 0$ as required.

\section{Positivity in the case where there is quiescence}

Using a minor adaptation of our previous results, it is easy to see that, for $\phi \in X_{\alpha}$, the system of equations

$$
\hat{p}(t)=S(t-\sigma) \phi+\int_{\sigma}^{t} S(t-\hat{\tau}) K\left(f(\hat{\tau}), A^{\alpha} \hat{p}(\hat{\tau})\right) \mathrm{d} \hat{\tau}
$$


has a unique solution $\hat{p} \in C\left([\sigma, \infty) ; X_{\alpha}\right)$. The solution is positive if $\phi \in X_{\alpha,+}=$ $\Pi_{i=0}^{n} L_{+}^{1}\left(\left(0, a_{1}\right) \times\left(0, s_{1}\right) ; D\left(A_{i}^{\alpha}\right)\right)$. Thus the solutions generate a positive evolution system $U(t, \sigma)$ : $X_{\alpha} \rightarrow X_{\alpha}$, such that for all $t \geq \sigma \geq \tau \geq 0$, and all $\phi \in X_{\alpha}, U(t, \sigma) U(\sigma, \tau) \phi=U(t, \tau) \phi$. We can adapt Lemma 1.1 of [21] to show that for $\gamma>\hat{\omega}$ and large enough there exists $m_{\gamma}>0$, independent of $\sigma$, such that for all $\phi \in X_{\alpha}$

$$
\|U(t, \sigma) \phi\|_{X_{\alpha}} \leq m_{\gamma} e^{\gamma(t-\sigma)}\|\phi\|_{X_{\alpha}} .
$$

Thus there is continuous dependence on the initial data and $(t, \sigma) \rightarrow U(t, \sigma) \phi$ is continuous.

Lemma 10. Take $h \in C\left([0, \infty) ; X_{\alpha}\right)$. Then $\check{p}$ satisfies

$$
\check{p}(t)=S(t) \phi+\int_{0}^{t} S(t-\hat{\tau})\left(K\left(f(\hat{\tau}), A^{\alpha} \check{p}(\hat{\tau})\right)+h(\hat{\tau})\right) \mathrm{d} \hat{\tau}
$$

if and only if

$$
\check{p}(t)=U(t, 0) \phi+\int_{0}^{t} U(t, \hat{\tau}) h(\hat{\tau}) \mathrm{d} \hat{\tau} .
$$

Proof. Note that $\hat{p}(t)=U(t, \sigma) \phi$ satisfies (8.1).

Suppose first that $\check{p}$ satisfies (8.2). We show that if

$$
w(t)=U(t, 0) \phi+\int_{0}^{t} U(t, \hat{\sigma}) h(\hat{\sigma}) \mathrm{d} \hat{\sigma},
$$

then $w$ satisfies (8.2) so that, by uniqueness of solutions, $w=\check{p}$ as required. The converse is then immediate. But

$$
\begin{aligned}
& w(t)=S(t) \phi+\int_{0}^{t} S(t-\sigma) K\left(f(\sigma), A^{\alpha} U(\sigma, 0) \phi\right) \mathrm{d} \sigma \\
& +\int_{0}^{t} S(t-\hat{\sigma}) h(\hat{\sigma}) \mathrm{d} \hat{\sigma}+\int_{0}^{t} \int_{\hat{\tau}}^{t} S(t-\hat{\sigma}) K\left(f(\hat{\sigma}), A^{\alpha} U(\hat{\sigma}, \hat{\tau}) h(\hat{\tau})\right) \mathrm{d} \hat{\sigma} \mathrm{d} \hat{\tau} \\
& =S(t) \phi+\int_{0}^{t} S(t-\sigma) h(\sigma) \mathrm{d} \sigma \\
& +\int_{0}^{t} S(t-\sigma)\left\{K\left(f(\sigma), A^{\alpha} U(\sigma, 0) \phi+\int_{0}^{\sigma} K\left(f(\sigma), A^{\alpha} U(\sigma, \hat{\tau}) h(\hat{\tau})\right) \mathrm{d} \hat{\tau}\right\} \mathrm{d} \sigma\right. \\
& =S(t) \phi+\int_{0}^{t} S(t-\sigma) h(\sigma) \mathrm{d} \sigma+\int_{0}^{t} S(t-\sigma) K\left(f(\sigma), A^{\alpha} w(\sigma)\right) \mathrm{d} \sigma,
\end{aligned}
$$

so $w$ satisfies (8.2) and the result is proved.

We now assume that

$\mathrm{H}(8 . .1)$ (a) $\phi \geq 0, \zeta \geq 0$; (b) $f_{0} \geq 0, m_{0} \geq 0, w_{0} \geq 0$.

$\mathrm{H}(8 . .2)$ (a) $\tau_{i}, \sigma_{i}, \nu_{i}$ are all independent of $x$; (b) $\sigma_{i} \geq 0, \tau_{i} \geq 0$ 
$\mathrm{H}(8 . .3) \rho_{m} \geq 0, \beta_{w} \geq 0$

Suppose that $\mathrm{H}(8 . .2)$ (a) holds and $\zeta \in D\left(A^{\alpha}\right)$. Thus, if $\tau$ is the matrix with $\tau_{i}$ on the diagonal and zero elsewhere, $\tau q \in C\left([0, \infty) ; X_{\alpha}\right)$, and

$$
p(t)=U(t, 0) \phi+\int_{0}^{t} U(t, \hat{\sigma}) \tau(w(\hat{\sigma}), N(\hat{\sigma})) q(\hat{\sigma}) \mathrm{d} \hat{\sigma} .
$$

It now follows that as $U(t, \sigma) \phi$ satisfies (6.15) and (6.16), for $t>0, p$ also satisfies (6.15) and (6.16). That is $p$ satisfies the boundary conditions (2.6) and (2.7) (for $t>0$ ).

Theorem 11. Suppose that hypotheses $H(3 . .1), H(4 . .1), H(4 . .2), H(5 . .1), H(6 . .2), H(6 . .3), H(7 . .2)$, $H(8 . .1)(a)$ and $H(8 . .2)$ are satisfied, that $\frac{1}{2}<\alpha<1$ and $\left[f_{0}, m_{0}, w_{0}, \phi, \zeta\right]^{t} \in D\left(A_{f}\right) \times L^{1}(\Omega)^{2} \times X_{\alpha}^{2}$. Then the solutions of (4.4)-(4.5) are positive. If in addition $H(8 . .1)(b)$ and $H(8 . .3)$ also hold then solutions of (4.1)-(4.3) are also positive.

Proof. We set up an iteration

$$
\begin{aligned}
p^{0}(t)= & U(t, 0) \phi \\
q_{i}^{0}(t)= & \exp -\int_{0}^{t}\left(\nu_{i}(w(\hat{\tau}), N(\hat{\tau}))+\tau_{i}(w(\hat{\tau}), N(\hat{\tau}))\right) \mathrm{d} \hat{\tau} \zeta_{i} \\
p^{m}(t)= & U(t, 0) \phi+\int_{0}^{t} U(t, \hat{\tau}) \tau(w(\hat{\tau}), N(\hat{\tau})) q^{m-1}(\hat{\tau}) \mathrm{d} \hat{\tau} \\
q_{i}^{m}(t)= & \exp -\int_{0}^{t}\left(\nu_{i}(w(\hat{\tau}), N(\hat{\tau}))+\tau_{i}(w(\hat{\tau}), N(\hat{\tau}))\right) \mathrm{d} \hat{\tau} \zeta_{i} \\
& +\int_{0}^{t} \exp -\int_{\hat{\sigma}}^{t}\left(\nu_{i}(w(\hat{\tau}), N(\hat{\tau}))+\tau_{i}(w(\hat{\tau}), N(\hat{\tau}))\right) \mathrm{d} \hat{\tau} \sigma_{i}(w(\hat{\sigma})) p_{i}^{m-1}(\hat{\sigma}) \mathrm{d} \hat{\sigma} .
\end{aligned}
$$

Then $p^{0} \in C\left([0, \infty) ; X_{\alpha}\right), p^{0} \geq 0$ and $q^{0} \in C\left([0, \infty) ; X_{\alpha}\right)$ and $q^{0} \geq 0$, so by induction $p^{m} \in C\left([0, \infty) ; X_{\alpha}\right), p^{m} \geq 0$ and $q^{m} \in C\left([0, \infty) ; X_{\alpha}\right), q^{m} \geq 0$. But by the usual convergence arguments $p^{m} \rightarrow p$, and $q^{m} \rightarrow q$. Hence, as in Theorem 9, $p \geq 0$ and $q \geq 0$. That $f, m$ and $w$ are also positive now follows as in [7], Theorem 9.

\section{Growth bound when there is no quiescence}

When there is no quiescence we can obtain a growth bound using a similar method to that used in [7].

Theorem 12. Suppose that there is no quiescence, that hypotheses $H(3 . .1), H(4 . .1), H(4 . .2)$, $H(5 . .1), H(6 . .1), H(6 . .2), H(6 . .3), H(7 . .1)$ and $H(7 . .2)$ hold and $\mu_{i}+\sigma_{i} \geq 0$ for all $i$. Then

$$
\left\|p_{i}(t)\right\|_{X} \leq c_{i}\left(\phi^{i}\right) e^{\left(\bar{\theta}_{i}-\omega\right) t}
$$


Further, for $t \geq \sigma \geq 0$,

$$
\|U(t, \sigma) \phi\|_{X^{n+1}} \leq M e^{\left(\bar{\theta}_{n}-\omega\right)(t-\sigma)}\|\phi\|_{X^{n+1}},
$$

where $c_{i}\left(\phi^{i}\right)$ and $M$ are as in Lemma 1.

Proof. Using the same argument as in [7] we see that from (6.8),

$$
\int_{\Omega} \tilde{p}_{i, c, d}(t) \mathrm{d} x \leq e^{-\omega\left(t-t_{c, d}\right)} \int_{\Omega} \tilde{p}_{i, c, d}\left(t_{c, d}\right) \mathrm{d} x .
$$

So that if $0<t<a$ and $0<t<s$

$$
\left\|p_{i}(a, s, t)\right\|_{1} \leq e^{-\omega t}\left\|\phi_{i}(a-t, s-t)\right\|_{1},
$$

while if $0 \leq a<t$ and $0 \leq a<s$

$$
\left\|p_{i}(a, s, t)\right\|_{1} \leq e^{-\omega a}\left\|p_{i}(0, s-a, t-a)\right\|_{1} .
$$

Set $k_{i}(t)=\int_{0}^{s_{1}}\left\|p_{i}(0, s, t)\right\|_{1} \mathrm{~d} s$. Then

$$
\left\|p_{i}(t)\right\|_{X} \leq e^{-\omega t}\left\|\phi_{i}\right\|_{X}+\int_{0}^{t} e^{-\omega a} k_{i}(t-a) \mathrm{d} a
$$

and hence

$$
e^{\omega t}\left\|p_{i}(t)\right\|_{X} \leq\left\|\phi_{i}\right\|_{X}+\int_{0}^{t} e^{\omega a} k_{i}(a) \mathrm{d} a
$$

Now from (6.15)

$$
e^{\omega t} k_{i}(t) \leq \bar{\theta}_{i} e^{\omega t}\left\|p_{i}(t)\right\|_{X}+\bar{\theta}_{i-1} \frac{\psi_{i-1}}{1-\psi_{i-1}} e^{\omega t}\left\|p_{i-1}(t)\right\|_{X}
$$

so using (9.3) $k_{i}$ satisfies the same equation (3.20) as $g_{i}$. Thus as in Lemma $1 k_{i}(t) \leq \bar{\theta}_{i} c_{i}\left(\phi^{i}\right) e^{\left(\bar{\theta}_{i}-\omega\right) t}$ so the result follows from (9.3).

If $\sigma=0$ then (9.2) follows immediately. Otherwise the estimates above can be adapted.

\section{Regularity and growth bound when there is quiescence}

In this section we assume that $a_{1}$ and $s_{1}$ are both infinite. Our previous results all hold as before provided we change hypothesis $\mathrm{H}(3 . .1)$ to (see [23])

$\mathrm{H}\left(3 . .1^{\prime}\right) \theta_{i} \in C\left([0, \infty) \times[0, \infty) \times[0, \infty) ; \mathbb{R}^{+}\right)$, and there exists $\bar{a}>0$ and $\bar{s}>0$ such that $\theta_{i}(\hat{a}, \hat{s}, s)=0$ for $\bar{a}<\hat{a}$ and $\bar{s}<\hat{s}, s$. If

$$
\bar{\theta}_{i}:=\sup _{\hat{a} \in[0, \infty), \hat{s} \in[0, \infty)} \int_{0}^{\infty} \theta_{i}(\hat{a}, \hat{s}, s) \mathrm{d} s,
$$


then $\bar{\theta}_{i}$ is strictly increasing with $i$.

We will assume that

$\mathrm{H}(10 . .1) \mu_{i}, \sigma_{i}, \tau_{i}, \nu_{i}$ are independent of $a$ and $s$.

First we consider an equation for $P_{i}$ and $Q_{i}$. Write

$$
\Phi=\int_{0}^{\infty} \int_{0}^{\infty} \phi(a, s) \mathrm{d} a \mathrm{~d} s, \quad Z=\int_{0}^{\infty} \int_{0}^{\infty} \zeta(a, s) \mathrm{d} a \mathrm{~d} s
$$

and

$$
I_{i}(t)=\int_{0}^{\infty} \int_{0}^{\infty} \int_{0}^{\infty}\left(\theta_{i}(\hat{a}, \hat{s}, s) p_{i}(\hat{a}, \hat{s}, t)+\frac{\psi_{i-1}}{1-\psi_{i-1}} \theta_{i-1}(\hat{a}, \hat{s}, s) p_{i-1}(\hat{a}, \hat{s}, t)\right) \mathrm{d} \hat{s} \mathrm{~d} \hat{a} \mathrm{~d} s .
$$

Note that

$$
\left\|I_{i}(t)\right\|_{1} \leq \bar{\theta}_{i}\left\|P_{i}(t)\right\|_{1}+\frac{\psi_{i-1}}{1-\psi_{i-1}} \bar{\theta}_{i-1}\left\|P_{i-1}(t)\right\|_{1} .
$$

For convenience we will write

$$
\begin{aligned}
& L_{i}\left(p_{i}(t), q_{i}(t)\right)= \\
& \quad-G_{i}\left(f(t), A_{i}^{\alpha} p_{i}(t)\right)-\left(\mu_{i}(w(t), N(t))+\sigma_{i}(w(t), N(t))\right) p_{i}(t)+\tau_{i}(w(t), N(t)) q_{i}(t),
\end{aligned}
$$

and $L^{i}\left(p^{i}(t), q^{i}(t)\right)=\left[L_{0}\left(p_{0}(t), q_{0}(t)\right), \ldots, L_{i}\left(p_{i}(t), q_{i}(t)\right)\right]^{t}$; and similarly for $L_{i}\left(P_{i}(t), Q_{i}(t)\right)$.

Theorem 13. Suppose that hypotheses $H\left(3 . .1^{\prime}\right), H(4 . .1), H(4 . .2), H(5 . .1)$ and $H(10 . .1)$ hold, and $\left[f_{0}, m_{0}, w_{0}, \phi, \zeta\right]^{t} \in D\left(A_{f}\right) \times L^{1}(\Omega)^{2} \times X_{\alpha} \times X^{n+1}$ where $\frac{1}{2}<\alpha<1$. Then $P_{i}, Q_{i}$ satisfy

$$
\begin{aligned}
P_{i}(t) & =T_{i}(t) \Phi_{i}+\int_{0}^{t} T_{i}(t-\hat{\tau})\left(L_{i}\left(P_{i}(\hat{\tau}), Q_{i}(\hat{\tau})\right)+I_{i}(\hat{\tau})\right) \mathrm{d} \hat{\tau} \\
Q_{i}(t) & =\exp -\int_{0}^{t}\left(\nu_{i}(w(\hat{\tau}), N(\hat{\tau}))+\tau_{i}(w(\hat{\tau}), N(\hat{\tau}))\right) \mathrm{d} \hat{\tau} Z_{i}+ \\
& \int_{0}^{t} \exp -\int_{\hat{\sigma}}^{t}\left(\nu_{i}(w(\hat{\tau}), N(\hat{\tau}))+\tau_{i}(w(\hat{\tau}), N(\hat{\tau}))\right) \mathrm{d} \hat{\tau} \sigma_{i}(w(\hat{\sigma}), N(\hat{\sigma})) P_{i}(\hat{\sigma}) \mathrm{d} \hat{\sigma}
\end{aligned}
$$

and these equations with (4.1)-(4.3) have a unique classical solution $[f, m, w, P, Q]^{t}$ in the sense that:

$f, m, w$ satisfy (6.18)-(6.21) (with $\mu_{w}$ depending also on $P$ and $\left.Q\right)$, and (2.10);

$P_{i}, Q_{i} \in C\left([0, \infty) ; L^{1}(\Omega)\right) \cap C^{1}\left((0, \infty) ; L^{1}(\Omega)\right)$, and for $t>0 P_{i}(t) \in D\left(A_{i}\right)$, and $P_{i}$ and $Q_{i}$ satisfy

$$
\begin{aligned}
& \frac{\mathrm{d}}{\mathrm{d} t} P_{i}(t)= \delta_{i} \Delta P_{i}(t)-\omega P_{i}(t)-\chi_{i} \nabla \cdot\left(P_{i}(t) \nabla f(t)\right)- \\
&\left.\left(\mu_{i}(x, w(t), N(t))+\sigma_{i}(w(t), N(t))\right) P_{i}(t)+\tau_{i}(w(t), N(t)) Q_{i}(t)+I_{i}(t)\right)(10.5) \\
& \frac{\mathrm{d}}{\mathrm{d} t} Q_{i}(t)=-\nu_{i}(w(t), N(t)) Q_{i}(t)+\sigma_{i}(w(t), N(t)) P_{i}(t)-\tau_{i}(w(t), N(t)) Q_{i}(t), \quad(10.6) \\
& P(0)=\Phi \text { and } Q(0)=Z .
\end{aligned}
$$


Proof. To show that (10.3) holds, we note first that from (4.4) and (3.12)

$$
I_{i}(t)=\int_{0}^{\infty}\left(\left(S_{i}(t) \phi^{i}\right)(0, s)+\int_{0}^{t}\left(S_{i}(t-\hat{\tau}) L^{i}\left(p^{i}(\hat{\tau}), q^{i}(\hat{\tau})\right)\right)(0, s) \mathrm{d} \hat{\tau}\right) \mathrm{d} s .
$$

Also, using (3.8) and (3.10),

$$
\begin{aligned}
\int_{0}^{\infty} & \int_{0}^{\infty} \int_{0}^{t}\left(S_{i}(t-\hat{\tau}) L^{i}\left(p^{i}(\hat{\tau}), q^{i}(\hat{\tau})\right)\right)(a, s) \mathrm{d} \hat{\tau} \mathrm{d} a \mathrm{~d} s \\
& \left.=\int_{0}^{t} T_{i}(t-\hat{\tau}) \int_{0}^{\infty} \int_{0}^{\infty} L_{i}\left(p_{i}(\hat{\tau}), q_{i}(\hat{\tau})\right)\right)(a, s) \mathrm{d} a \mathrm{~d} s \mathrm{~d} \hat{\tau} \\
& +\int_{0}^{t} \int_{0}^{t-\sigma} \int_{\hat{a}}^{\infty} T_{i}(\hat{a})\left(S_{i}(t-\sigma-\hat{a}) L^{i}\left(p^{i}(\sigma), q^{i}(\sigma)\right)\right)(0, \hat{s}-\hat{a}) \mathrm{d} \hat{s} \mathrm{~d} \hat{a} \mathrm{~d} \sigma \\
& =\int_{0}^{t} T_{i}(t-\hat{\tau}) L_{i}\left(P_{i}(\hat{\tau}), Q_{i}(\hat{\tau})\right) \mathrm{d} \hat{\tau} \\
& +\int_{0}^{t} T_{i}(t-\hat{\tau}) \int_{0}^{\infty} \int_{0}^{\hat{\tau}}\left(S_{i}(\hat{\tau}-\sigma) L^{i}\left(p^{i}(\sigma), q^{i}(\sigma)\right)\right)(0, \hat{\sigma}) \mathrm{d} \sigma \mathrm{d} \hat{\sigma} \mathrm{d} \hat{\tau}
\end{aligned}
$$

Thus, integrating (4.4) with respect to $a$ and $s$ we get

$$
\begin{aligned}
P_{i}(t)= & T_{i}(t) \Phi_{i}+\int_{0}^{t} T_{i}(t-\hat{\tau})\left(L_{i}\left(P_{i}(\hat{\tau}), Q_{i}(\hat{\tau})\right)+\right. \\
& \left.\int_{0}^{\infty}\left(\left(S_{i}(\hat{\tau}) \phi^{i}\right)(0, s)+\int_{0}^{\hat{\tau}}\left(S_{i}(\hat{\tau}-\sigma) L^{i}\left(p^{i}(\sigma), q^{i}(\sigma)\right)\right)(0, s) \mathrm{d} \sigma\right) \mathrm{d} s\right) \mathrm{d} \hat{\tau},
\end{aligned}
$$

and (10.3) follows immediately. (10.4) is trivial.

That $f$ is a classical solution of (4.1) follows as in Theorem 8. Also, in the same way as before, for any $T>0, Q_{i} \in C^{1}\left([0, T] ; L^{1}(\Omega)\right)$, so that $Q_{i}$ is Lipschitz continuous on $[0, T]$ and is a classical solution of (10.4). Also $w(t)$ is locally Hölder continuous. Operate on (10.3) with $A_{i}^{\alpha}$ to get an equation for $V_{i}$,

$$
V_{i}(t)=T_{i}(t) A_{i}^{\alpha} \Phi_{i}+\int_{0}^{t} A_{i}^{\alpha} T_{i}(t-\hat{\tau})\left(L_{i}\left(A_{i}^{-\alpha} V_{i}(\hat{\tau}), Q_{i}(\hat{\tau})\right)+I_{i}(\hat{\tau})\right) \mathrm{d} \hat{\tau}
$$

For each i, $V_{i}, P_{i}, Q_{i} \in C\left([0, \infty) ; L^{1}(\Omega)\right)$ so, using Lemma $2, L_{i}\left(A_{i}^{-\alpha} V_{i}, Q_{i}\right)+I_{i}$ $\in C\left([0, \infty) ; L^{1}(\Omega)\right)$. Thus as in [13] Theorem 6.3.1 $V_{i}$ (and hence also $P_{i}$ ) is locally Hölder continuous. Thus $L_{i}\left(A_{i}^{-\alpha} V_{i}, Q_{i}\right)+I_{i}$ is locally Hölder continuous and $P_{i}$ is a classical solution of (10.3). That $m$ and $w$ are classical solution of (4.2) and (4.3) follows from the Hölder continuity of $f, w, P_{i}, Q_{i}$.

Remark. Thus, when $a_{1}$ and $s_{1}$ are infinite and the coefficients are independent of $a$ and $s, P_{i}(t)$ is locally Hölder continuous so, as also $Q_{i}(t)$ is Lipschitz continuous, Proposition 7 and Theorems 8,9 , and 10 now all hold without hypothesis $\mathrm{H}(6 . .3)$. 
We now look at growth bounds on $P(t)+Q(t)$. The bounds depend on the relative magnitudes of the various coefficients and we only give an example of such a result. We use equations (10.5) and (10.6). The advantage of doing this is that when we add these equations the terms involving $\sigma_{i}$ and $\tau_{i}$ cancel. We consider the case where $P$ and $Q$ are positive. Set

$$
v_{i}=\max \left\{\bar{\theta}_{i}-\omega-\inf \mu_{i},-\inf \nu_{i}\right\},
$$

and assume

$\mathrm{H}(10 . .2) v_{i}$ is strictly increasing with $i$.

Theorem 14. Suppose that hypotheses $H\left(3 . .1^{\prime}\right), H(4 . .1), H(4 . .2), H(5 . .1), H(7 . .2), H(8 . .1)(a)$, $H(8 . .2), H(10 . .1)$ and $H(10 . .2)$ are satisfied, that $\frac{1}{2}<\alpha<1$, and $\left[f_{0}, m_{0}, w_{0}, \phi, \zeta\right]^{t} \in D\left(A_{f}\right) \times$ $L^{1}(\Omega)^{2} \times X_{\alpha}^{2}$. Then

$$
\left\|P_{i}(t)+Q_{i}(t)\right\|_{1} \leq d_{i}\left(\phi^{i}+\zeta^{i}\right) e^{v_{i} t}, i \geq 0,
$$

where $d_{0}\left(\phi^{0}\right)=\left\|\phi_{0}\right\|_{X}$ and for $i \geq 1$,

$$
d_{i}\left(\phi^{i}\right)=\left\|\phi_{i}\right\|_{X}+\sum_{k=0}^{i-1}\left\{\Pi_{j=k}^{i-1} \frac{\psi_{j} \bar{\theta}_{j}}{\left(1-\psi_{j}\right)\left(v_{j+1}-v_{j}\right)}\right\}\left\|\phi_{k}\right\|_{X} .
$$

Proof. Note first that $p \geq 0$ and $q \geq 0$. We integrate equations (10.5) and (10.6) over $\Omega$ and add to get

$$
\begin{aligned}
\frac{\mathrm{d}}{\mathrm{d} t}\left\|P_{i}(t)+Q_{i}(t)\right\|_{1} & \leq\left(\bar{\theta}_{i}-\omega-\inf \mu_{i}\right)\left\|P_{i}(t)\right\|_{1}-\left(\inf \nu_{i}\right)\left\|Q_{i}(t)\right\|_{1}+\frac{\bar{\theta}_{i-1} \psi_{i-1}}{1-\psi_{i-1}}\left\|P_{i-1}(t)\right\|_{1} \\
& \leq v_{i}\left\|P_{i}(t)+Q_{i}(t)\right\|_{1}+\frac{\bar{\theta}_{i-1} \psi_{i-1}}{1-\psi_{i-1}}\left\|P_{i-1}(t)\right\|_{1} .
\end{aligned}
$$

Thus

$e^{-v_{i} t}\left\|P_{i}(t)+Q_{i}(t)\right\|_{1} \leq\left\|\phi_{i}+\zeta_{i}\right\|_{X}+\int_{0}^{t} e^{-\left(v_{i}-v_{i-1}\right) \sigma} \frac{\bar{\theta}_{i-1} \psi_{i-1}}{1-\psi_{i-1}} e^{-v_{i-1} \sigma}\left\|P_{i-1}(\sigma)+Q_{i-1}(\sigma)\right\|_{1} \mathrm{~d} \sigma$,

and the result follows by induction (c.f. Lemma 1).

\section{Numerical Examples}

We illustrate the theoretical results above with numerical examples. The parameters for the examples are chosen for illustrative purposes. For simplicity we consider a 1-dimensional spatial region $\Omega=(0,10)$ and we do not include the size variable $s$ nor quiescent cells. We consider a population of two tumor cell phenotypes $p_{0}(x, a, t)$ and $p_{1}(x, a, t)$ with age range $a \in[0,4]$.

Example 1. In this example there is no haptotaxis present $\left(\chi_{0}=0.0, \chi_{1}=0.0\right)$. The parameters for the extracellular matrix density $\mathrm{MM}$ are $\delta_{f}=0.0, \omega_{f}=0.0$, and the degradation coefficient is

$$
\lambda_{f}(x, m, w, P)=0.5 \lambda_{\epsilon}^{0}(x) \int_{0}^{10} \kappa_{\sigma}(x, \hat{x}) \lambda^{1}(m(\hat{x})) d \hat{x}
$$


with $\epsilon=1.0$ and $\sigma=0.1$. Here $\lambda_{\epsilon}^{0}(x)$ is the regularization with respect to Neumann boundary conditions satisfying $\lambda_{\epsilon}^{0}(x) \equiv 1$ on $[\epsilon, 10-\epsilon], \lambda_{\epsilon}^{0}(x)$ is the $4^{\text {th }}$ degree polynomial on $[0, \epsilon]$, which is 0 at 0,1 at $\epsilon$, has $1^{\text {st }}$ derivative 0 at 0 , and has $1^{\text {st }}$ and $2^{\text {nd }}$ derivative 0 at $\epsilon$, and $\lambda_{\epsilon}^{0}(x)$ is the $4^{\text {th }}$ degree polynomial on $[10-\epsilon, 10]$, which is 0 at 10,1 at $10-\epsilon$, has $1^{\text {st }}$ derivative 0 at 10 , and has $1^{s t}$ and $2^{\text {nd }}$ derivative 0 at $10-\epsilon$. Also, $\lambda^{1}(z)=z /(1.0+.01 z)$ and $\kappa_{\sigma}(x, \hat{x})$ is the regularization with respect to spatial derivatives given by the normal probability distribution

$$
\kappa_{\sigma}(x, \hat{x})=\frac{1}{\sqrt{2 \pi} \sigma} e^{-\frac{(x-\hat{x})^{2}}{2 \sigma^{2}}} .
$$

The parameters for the matrix degradation enzyme MDE concentration are $\delta_{m}=.01, \omega_{m}=$ 1.0 , and $\rho_{m}(x, P)=1.0$. The parameters for the oxygen concentration are $\delta_{w}=.01, \omega_{w}=0.0$, $\beta_{w}(x, f)=5.0$, and $\mu_{w}(x, w, P)=1.0+\frac{2.0}{1.0+P}$. The parameters for the $p_{0}$ phenotype are $\delta_{0}=.05, \omega=0.0, \chi_{0}=0.0, \mu_{0}(x, a, w, P)=\beta_{0}(a)+\max \left\{0.0,1.0-\frac{w}{1.0+w}\right\}$ with division modulus

$$
\beta_{0}(a)=\left\{\begin{array}{l}
0.0, a \leq 2.0 \text { or } a \geq 4.0 \\
7.0(a-2.0)(4.0-a) e^{-2.0(a-2.0)}+0.1,2.0<a<4.0
\end{array}\right.
$$

and $\psi_{0}=0.001$. The parameters for the $p_{1}$ phenotype are $\delta_{1}=.05, \omega=0.0, \chi_{1}=0.0$, $\mu_{1}(x, a, w, N)=\beta_{1}(a)+\max \left\{0.0,1.0-\frac{w}{1.0+w}\right\}$ with division modulus

$$
\beta_{1}(a)=\left\{\begin{array}{l}
0.0, a \leq 1.5 \text { or } a \geq 4.0 \\
6.0(a-1.5)(4.0-a) e^{-2.0(a-1.5)}+0.1,1.5<a<4.0
\end{array}\right.
$$

and $\psi_{1}=0.001$ (see Fig. 1).
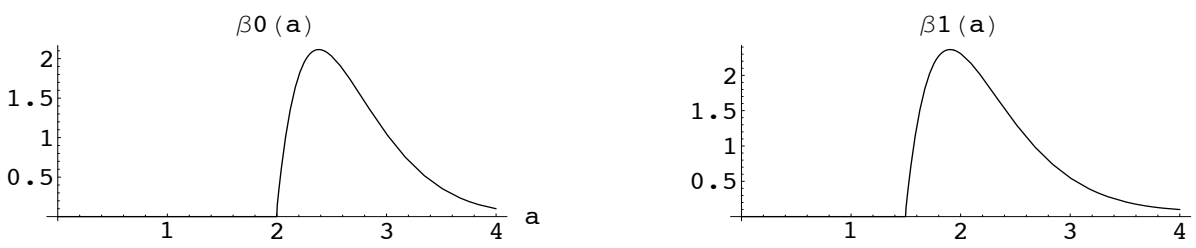

Figure 1: The division moduli $\beta_{0}(a)$ and $\beta_{1}(a)$ for the phenotypes $p_{0}$ and $p_{1}$. The average division cycle time (2.27) of phenotype $p_{1}$ is shorter than the average division cycle time (2.54) of $p_{0}$.

The initial values are $f(x, 0)=0.05 \cos \left(0.1 x^{2}\right)+.3, m(x, 0)=\hat{\eta}_{0}(x) \hat{\eta}_{1}(x), c(x, 0)=$ $4.0 f(x, 0), p_{0}(x, a, 0)=\hat{\xi}_{0}(a) \hat{\eta}_{0}(x), p_{1}(x, a, 0)=\hat{\xi}_{1}(a) \hat{\eta}_{1}(x)$ (see Fig. 2), with $\hat{\xi}_{0}(a)=2.0 e^{-a}$, $\hat{\xi}_{1}(a)=\left\{\begin{array}{l}10.0 a(1.0-a), 0.0 \leq a \leq 1.0 \\ 0.0, a>1.0\end{array} \hat{\eta}_{0}(x)=\left\{\begin{array}{l}0.0,0.0 \leq x \leq 3.5 \\ 10.0(x-3.5)^{2}(4.5-x)^{2}, 3.5<x<4.5 \\ 0.0, x \geq 4.5\end{array}\right.\right.$ 


$$
\hat{\eta}_{1}(x)=\left\{\begin{array}{l}
0.0,0.0 \leq x \leq 6.0 \\
5.0(x-6.0)^{2}(7.0-x)^{2}, 6.0<x<7.0 \\
0.0, x \geq 7.0
\end{array}\right.
$$
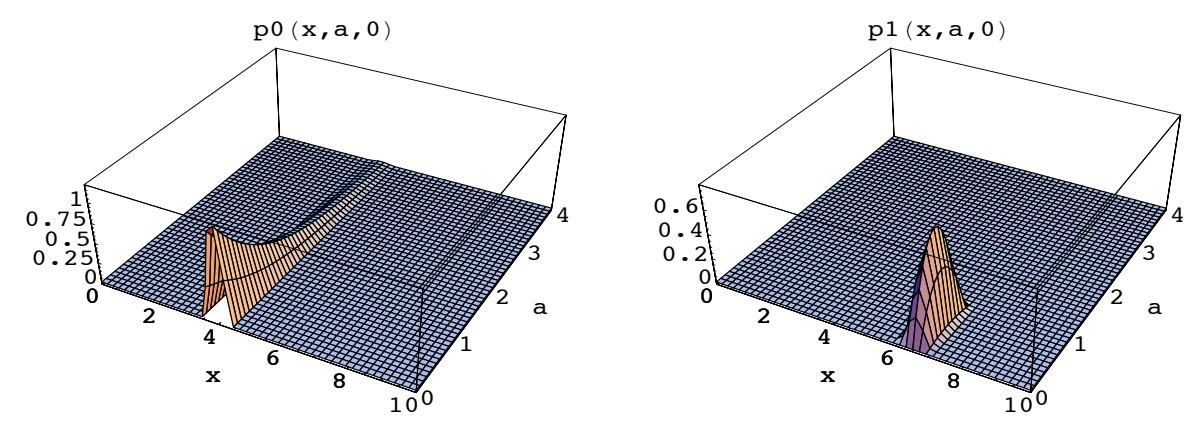

Figure 2: The spatial and age initial distributions of the phenotypes $p_{0}$ and $p_{1}$. At time $0, p_{0}$ consists of a larger number of cells located in the left side of the spatial domain and distributed through an extended range of cell ages, and $p_{1}$ consists of a smaller number of newly divided cells located on the right side of the spatial domain.

The simulation is shown in Fig. 3 (the space-age density plots at $t=0.0,1.5,3.0,4.5,6.0$ ), Fig. 4 (the graphs of $P_{0}(x, t)$ and $P_{1}(x, t)$ ), and Fig. 5 (the graphs of the total populations $\mathbf{P}_{0}(t)=$ $\int_{\Omega} \int_{0}^{a_{1}} p_{0}(x, a, t) \mathrm{d} a \mathrm{~d} x$, and $\left.\mathbf{P}_{1}(t)=\int_{\Omega} \int_{0}^{a_{1}} p_{1}(x, a, t) \mathrm{d} a \mathrm{~d} x\right)$. Both phenotype populations would ultimately extinguish as they degrade the extracellular matrix and exhaust the oxygen supply it produces. The spatial movement of the phenotype populations is due only to the random motility of cells.

Example 2. This example has the same parameters and initial values as Example 1 except that the haptotaxis parameters are $\chi_{0}=0.8$ for $p_{0}$ and $\chi_{1}=1.0$ for $p_{1}$. The phenotype $p_{1}$ thus possesses not only a shorter cell cycle contrasted with phenotype $p_{0}$, but a greater ability to move toward regions of extracellular matrix concentration, where there is a greater supply of oxygen. The advantage is demonstrated in a greater level of growth for phenotype $p_{1}$. The simulation is shown in Fig. 6, 7, and 8. The spatial movement of the phenotype cell populations is due to both random motility and haptotaxis. 
$t=0$

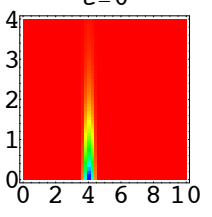

$t=0$

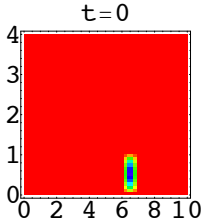

$t=1.5^{\prime}$

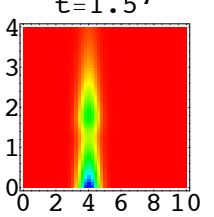

$t=1.5^{\prime}$

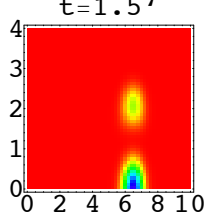

$t=3 .^{\prime}$

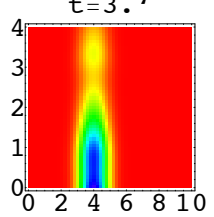

$t=3$.

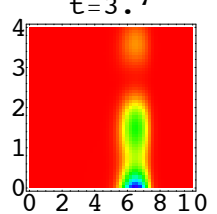

$t=4.5^{\prime}$

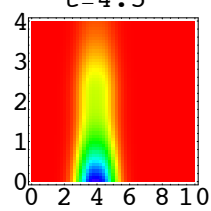

$t=4.5^{\prime}$

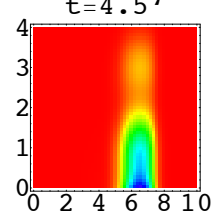

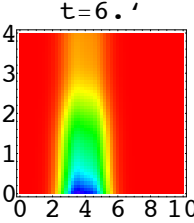

$t=6$,

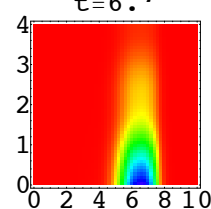

Figure 3: The space and age distributions of the phenotypes $p_{0}$ (top) and $p_{1}$ (bottom) at times $t=0.0,1.5,3.0,4.5,6.0$. The initial age synchronization of phenotype $p_{1}$ (with mostly newly divided cells) is dispersed over approximately 3 division cycles, and gradually resembles the age distribution of the unsynchronized phenotype $p_{0}$. The spatial distribution of both phenotypes is dispersed due to the random motility of cells.

\section{Concluding Remarks}

The model (2.1)-(2.10) incorporates many features of the complex biological processes involved in tumor growth, including individual cell growth and division, availability of essential nutrients such as oxygen, environmental influences such as extracellular macromolecules, transition to and from quiescence, and mutation to proliferatively advantaged cell phenotypes. The nonlinear partial differential equations describing these complex elements present many mathematical difficulties, particularly the terms describing the directed movement of cells in haptotaxis. We have developed a rigorous mathematical foundation for this model, and proved the global existence, uniqueness, positivity, continuous dependence, and regularity of solutions and obtained growth bounds. We have also provided a numerical example of the model in a special case to illustrate the importance of haptotaxis and phenotype mutation as contributing factors to tumor growth in spatial contexts. The full model (1)-(10) presents many challenges computationally, especially in 3-dimensional regions, with large numbers of phenotypes, and with realistic mutation rates. Further investigation is required to validate the model with scientifically supported parameters and establish its capacity to predict the evolution of a tumor.

\section{References}

[1] H. Amann. Dual semigroups and second order linear elliptic boundary value problems. Israel J. Math., 45 (1983), No. 2-3, 225-254.

[2] H. Amann. Linear and quasilinear problems, volume I: Abstract theory. Birkhauser, Basel 1995. 

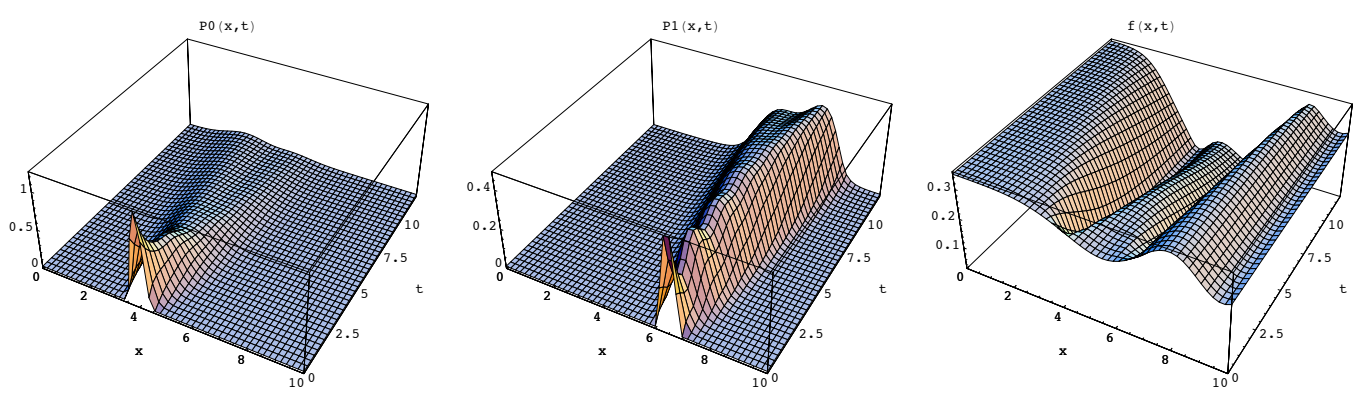

Figure 4: The spatial distributions of phenotypes $P_{0}(x, t)$ (left), $P_{1}(x, t)$ (middle), and extracellular matrix density $f(x, t)$ (right) as time evolves. Both phenotype populations disperse significantly from their initial spatial locations. Phenotype $p_{1}$ dominates $p_{0}$ in total growth, because of its higher proliferation rate.
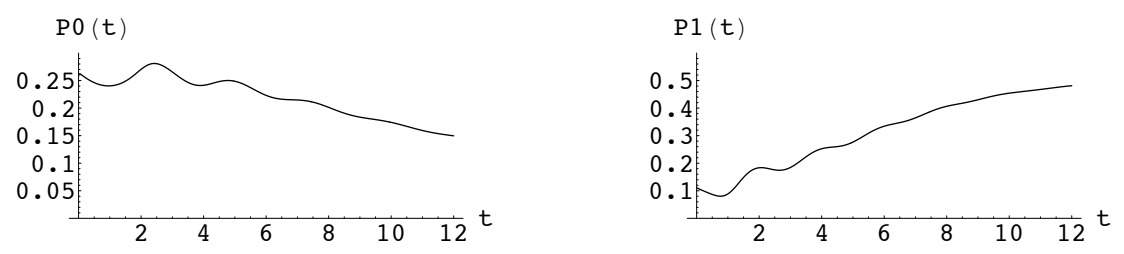

Figure 5: The total populations of phenotypes $\mathbf{P}_{0}(t)$ and $\mathbf{P}_{1}(t)$ as time evolves.

[3] A.R.A. Anderson. A hybrid mathematical model of solid tumour invasion: the importance of cell adhesion. Math. Med. Biol. IMA Journal, 22 (2005), 163-186.

[4] A.R.A. Anderson, A.M. Weaver, P.T. Cummings, V. Quaranta. Tumor morphology and phenotypic evolution driven by selective pressure from the microenvironment. Cell, 127 (2006), 905-915.

[5] B. Ayati, G.F. Webb, A.R.A. Anderson. Computational methods and results for structured multiscale models of tumor growth. Multi. Mod. Simul., 5 (2006), 1-20.

[6] J. Dyson, E. Sánchez, R. Villella-Bressan, G.F. Webb. An age and spatially structured model of tumor invasion with haptotaxis. Discr. Cont. Dyn. Sys.-B, 8 (2007), 45-60.

[7] J. Dyson, R. Villella-Bressan, G.F. Webb. An age and spatially structured model of tumor invasion with haptotaxis II. Mathematical Population Studies, 15 (2008), 73-95.

[8] P. Goméz-Mourelo, E. Sánchez, L. Casasús, G.F. Webb. A fully continuous individual-based model of tumor cell evolution. Submitted.

[9] C. Greenman, P. Stephens, R. Smith, et al. Patterns of somatic mutation in human cancer genomes. Science, 446 (2006), 153-158. 

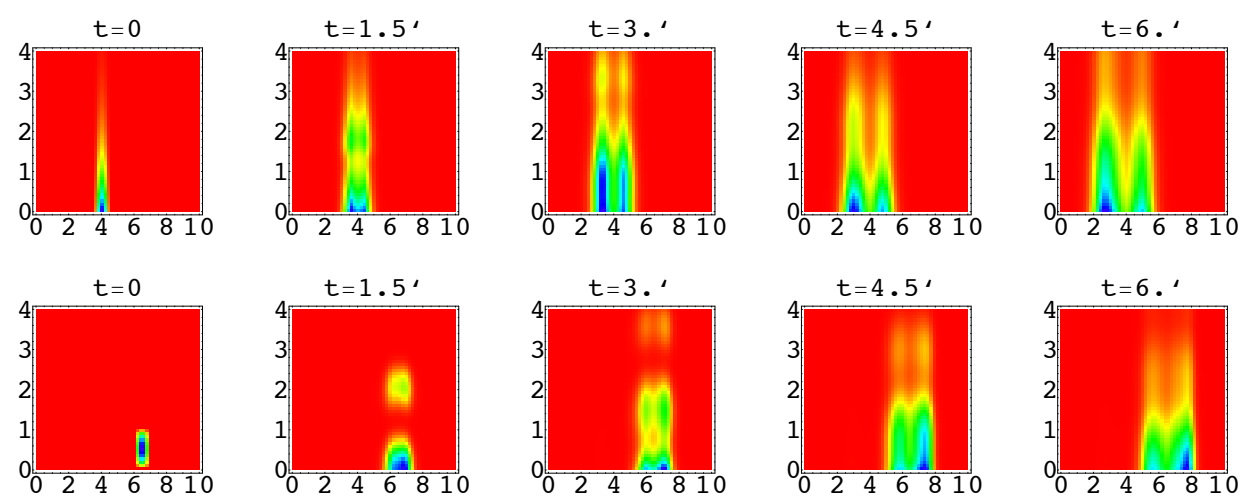

Figure 6: The space and age distributions of the phenotypes $p_{0}$ (top) and $p_{1}$ (bottom) at times $t=0.0,1.5,3.0,4.5,6.0$ with haptotaxis. The spatial distribution of both phenotypes is dispersed more over time than in Example 1.
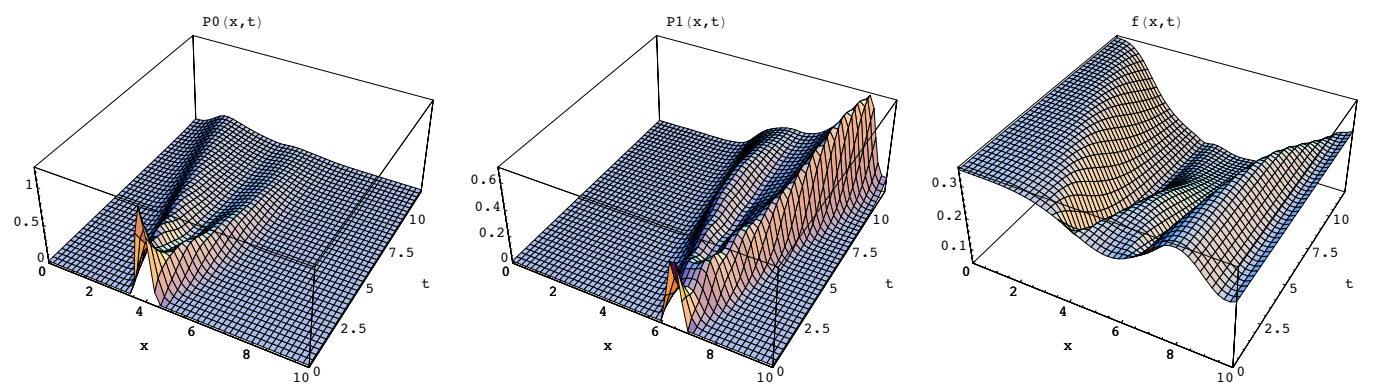

Figure 7: The spatial distributions of phenotypes $P_{0}(x, t)$ (left), $P_{1}(x, t)$ (middle), and extracellular matrix density $f(x, t)$ (right) with haptotaxis. More degradation of the extracellular matrix occurs than in Example 1.

[10] L. Lorenzi, A. Lunardi, G. Metafune, D. Pallara. Analytic semigroups and reaction diffusion problems. Internet Seminar, 2004-2005, Page 39.

[11] A. Lunardi. An introduction to interpolation theory. Internet Seminar, January 2005.

[12] K. Osaki, A. Yagi. Finite dimensional attractor for one-dimensional Keller-Segel equations. Funkcialaj Ekvacioj, 44 (2001), 441-469.

[13] A. Pazy. Semigroups of linear operators and applications to partial differential equations. Springer-Verlag, Berlin, 1983.

[14] H.B. Stewart. Generation of analytic semigroups by strongly elliptic operators. Trans. Amer. Math. Soc., 199 (1974), 141-162. 

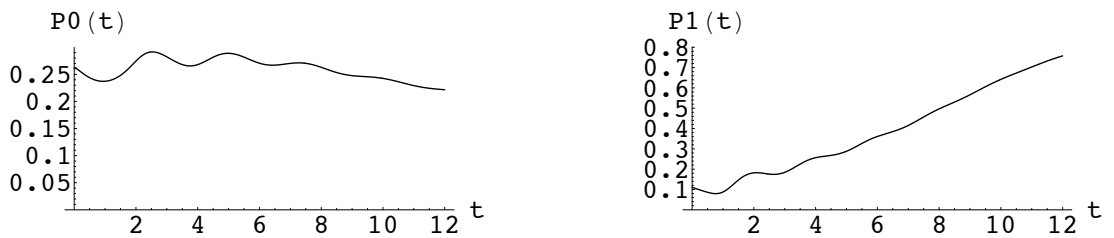

Figure 8: The total populations of phenotypes $\mathbf{P}_{0}(t)$ and $\mathbf{P}_{1}(t)$ with haptotaxis. Both phenotypes sustain higher population levels than in the case without haptotaxis in Example 1.

[15] H.B. Stewart. Generation of analytic semigroups by strongly elliptic operators under general boundary conditions. Trans. Amer. Math. Soc., 259 (1980), 299-310.

[16] H. Triebel. Interpolation theory, function spaces, differential operators. North-Holland Pub. Co., Amsterdam, 1978.

[17] H. Triebel. Theory of function spaces. Mathematik und ihre Anwendungen in Physik und Technik [Mathematics and its Applications in Physics and Technology], 38. Akademische Verlagsgesellschaft Geest \& Portig K.-G., Leipzig, 1983.

[18] Ch. Walker. Global well-posedness of a haptotaxis model with spatial and age structure. Diff. Int. Eq. 20, No. 9 (2007), 1053-1074.

[19] Ch. Walker. Global existence for an age and spatially structured haptotaxis model with nonlinear age-boundary conditions. Europ. J. Appl. Math. 19 (2008), 113-147.

[20] Ch. Walker, G.F. Webb. Global existence of classical solutions for a haptotaxis model. SIAM J. Math. Anal., 38 (2007), 1694-1712.

[21] G.F. Webb. Exponential representation of solutions to an abstract semi-linear differential equation. Pacific J. Math. 70 (1977), 269-279.

[22] G.F. Webb. Theory of nonlinear age-dependent population dynamics. Monographs and textbooks in Pure and Applied Mathematics, Marcel Dekker, Inc., 1985.

[23] G.F. Webb. Population models structured by age, size and spatial position. in Structured Population Models in Biology and Epidemiology, Lecture Notes in Mathematics, No. 1936, Mathematical Biosciences Series, P. Magal and S. Ruan, Eds., 2008, 1-49. 